\title{
Planar chiral analogues of PRODAN based on a [2.2]paracyclophane scaffold: Synthesis and photophysical studies
}

Simon Felder, ${ }^{a}$ Marie-Léonie Delcourt, ${ }^{a}$ Manon H. E. Bousquet, ${ }^{b}$ Denis Jacquemin, *,b Rafael Rodríguez, ${ }^{\mathrm{c}}$ Ludovic Favereau, ${ }^{\mathrm{c}}$ Jeanne Crassous, ${ }^{\mathrm{c}}$ Laurent Micouin*,a and Erica Benedetti*,a

(a) Université de Paris, CNRS, Laboratoire de Chimie et de Biochimie Pharmacologiques et Toxicologiques, F-75006 Paris, France

E-mail : erica.benedetti@parisdescartes.fr; laurent.micouin@parisdescartes.fr

(b) Université de Nantes, CNRS, CEISAM ULR 6230, F-44000 Nantes Cedex 3, France.

E-mail : Denis.Jacquemin@univ-nantes.fr.

(c) Univ Rennes, Institut des Sciences Chimiques de Rennes, UMR 6226 CNRS, Campus de Beaulieu, 35042 Rennes Cedex, France.

E-mail : jeanne.crassous@univ-rennes1.fr

\section{Supporting Information}




\section{Table of Contents}

HPCL chromatograms

Unpolarised spectroscopic studies

Theoretical investigations

Chiroptical properties

${ }^{1} \mathrm{H}$ NMR and ${ }^{13} \mathrm{C}$ NMR spectra 


\section{HPLC chromatograms}

4-formyl-16-hydroxymethyl[2.2]paracyclophane 6<smiles>O=Cc1cc2ccc1CCc1cccc(c1)CC2</smiles>

Chiral HPLC analysis: Column $=$ Chiralcel OD $(250$ x 4,6 mm); eluent $=n$-heptane $/ i-\mathrm{PrOH}(8: 2)$; flow $=1 \mathrm{~mL} / \mathrm{min} ; \lambda=210 \mathrm{~nm}, 254 \mathrm{~nm}$ or $300 \mathrm{~nm}$ ]

$\mathrm{HPLC}$ of racemic $( \pm)-6: \mathrm{tR}_{1}=19.9 \mathrm{~min} ; \mathrm{tR}_{2}=31.9 \mathrm{~min}$

$\mathrm{mAU}$

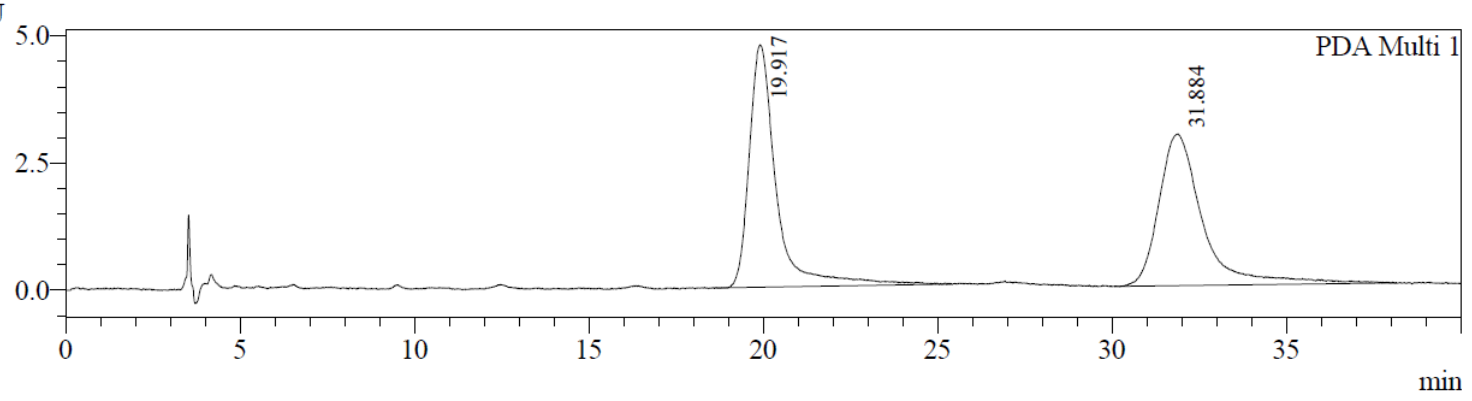

PDA Ch1 300nm 4nm

\begin{tabular}{|r|r|r|r|}
\hline \multicolumn{1}{|c|}{ Peak\# } & Ret. Time & \multicolumn{1}{c|}{ Area } & \multicolumn{1}{c|}{ Area \% } \\
\hline 1 & 19.917 & 259047 & 49.875 \\
\hline 2 & 31.884 & 260348 & 50.125 \\
\hline Total & & 519394 & 100.000 \\
\hline
\end{tabular}

HPLC of pure $\left(S_{\mathrm{p}}\right)-\mathbf{6}: \mathrm{tR}_{1}=20.9 \mathrm{~min} ; \mathrm{tR}_{2}=32.8 \mathrm{~min}, 97 \%$ ee

$\mathrm{mAU}$

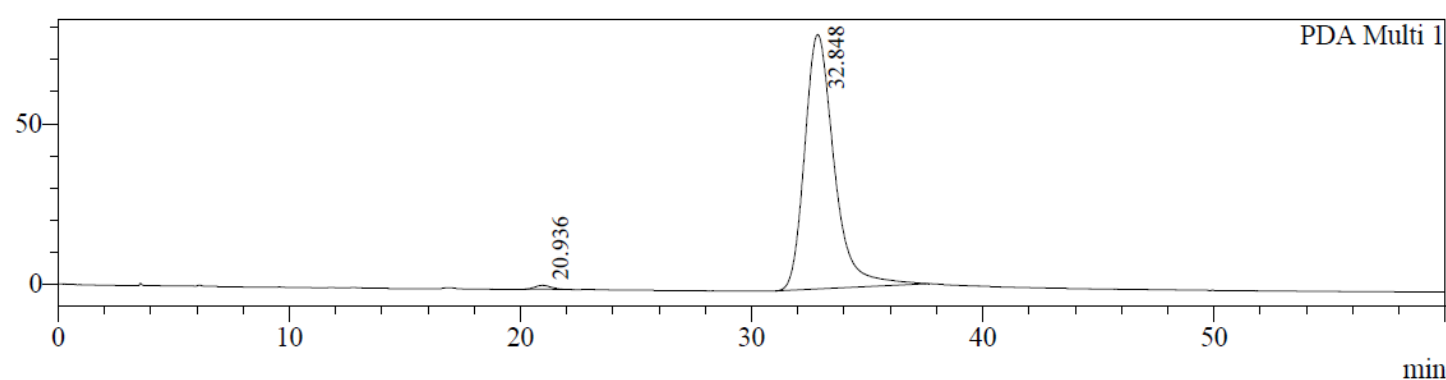

PDA Ch1 254nm 4nm

\begin{tabular}{|r|r|r|r|r|r|}
\hline \multicolumn{1}{|c|}{ Peak\# } & Ret. Time & \multicolumn{1}{c|}{ Area } & Height & \multicolumn{1}{|c|}{ Area \% } & Height \% \\
\hline 1 & 20.936 & 58698 & 1222 & 0.824 & 1.518 \\
\hline 2 & 32.848 & 7067046 & 79299 & 99.176 & 98.482 \\
\hline Total & & 7125744 & 80521 & 100.000 & 100.000 \\
\hline
\end{tabular}

HPLC of pure $\left(R_{\mathrm{p}}\right)-6: \mathrm{tR}_{1}=20.8 \mathrm{~min} ; \mathrm{tR}_{2}=32.8 \mathrm{~min}, 98 \%$ ee

mAU

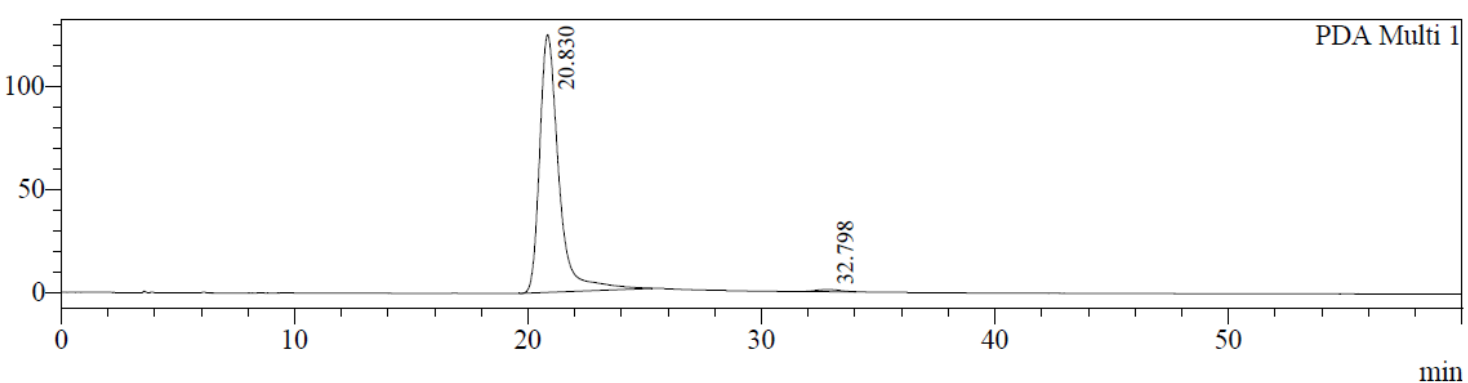

PDA Ch1 254nm 4nm

\begin{tabular}{|r|r|r|r|r|r|}
\hline \multicolumn{1}{|c|}{ Peak\# } & Ret. Time & \multicolumn{1}{c|}{ Area } & \multicolumn{1}{c|}{ Height } & \multicolumn{1}{c|}{ Area \% } & Height \% \\
\hline 1 & 20.830 & 7124023 & 125093 & 99.102 & 99.247 \\
\hline 2 & 32.798 & 64570 & 949 & 0.898 & 0.753 \\
\hline Total & & 7188593 & 126042 & 100.000 & 100.000 \\
\hline
\end{tabular}


1-[14-(Dimethylamino)tricyclo[8.2.2.2^\{4,7\}]hexadecal(12),4,6,10,13, 15-hexaen-5-yl]propan-1-one 4

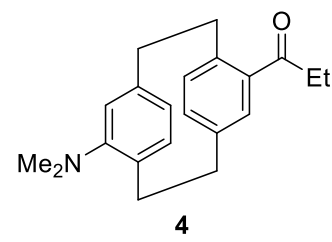

Chiral HPLC analysis: [Column $=$ Chiralcel AD $(250 \times 4,6 \mathrm{~mm})$; eluent $=n$-heptane $/ i-\mathrm{PrOH}(95: 5)$; flow $=0.5 \mathrm{~mL} / \mathrm{min} ; \lambda=254 \mathrm{~nm}]$

HPLC of racemic $( \pm)-4: \mathrm{tR}_{1}=8.4 \mathrm{~min} ; \mathrm{tR}_{2}=9.6 \mathrm{~min}$

mAU

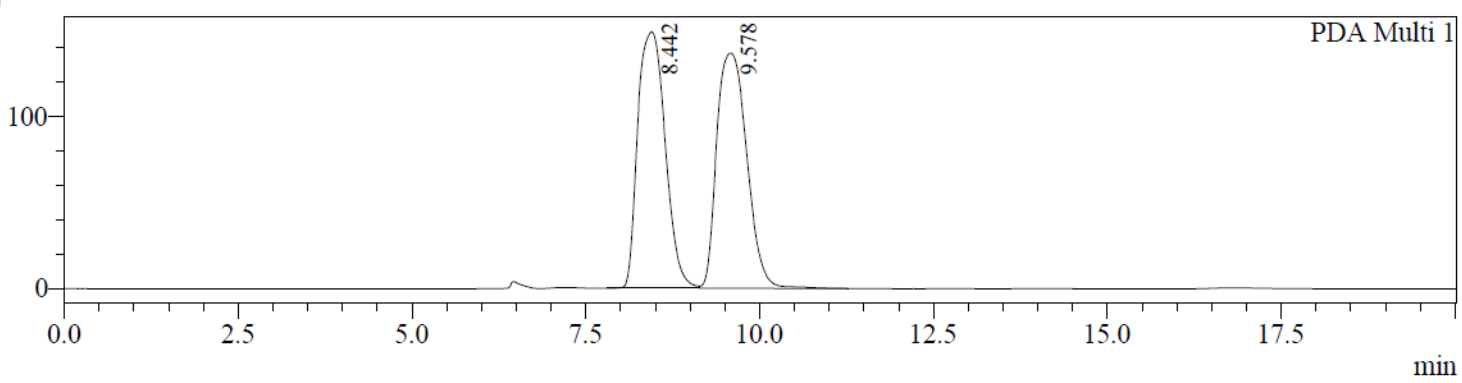

PDA Ch1 254nm 4nm

\begin{tabular}{|r|r|r|r|r|r|}
\hline \multicolumn{1}{|c|}{ Peak\# } & Ret. Time & \multicolumn{1}{c|}{ Area } & Height & \multicolumn{1}{c|}{ Area \% } & Height \% \\
\hline 1 & 8.442 & 4090522 & 148889 & 49.873 & 52.173 \\
\hline 2 & 9.578 & 4111422 & 136487 & 50.127 & 47.827 \\
\hline Total & & 8201944 & 285376 & 100.000 & 100.000 \\
\hline
\end{tabular}

HPLC of pure $\left(R_{\mathrm{p}}\right)-4: \mathrm{tR}_{1}=8.2 \mathrm{~min} ; \mathrm{tR}_{2}=9.4 \mathrm{~min}, 99 \%$ ee

mAU

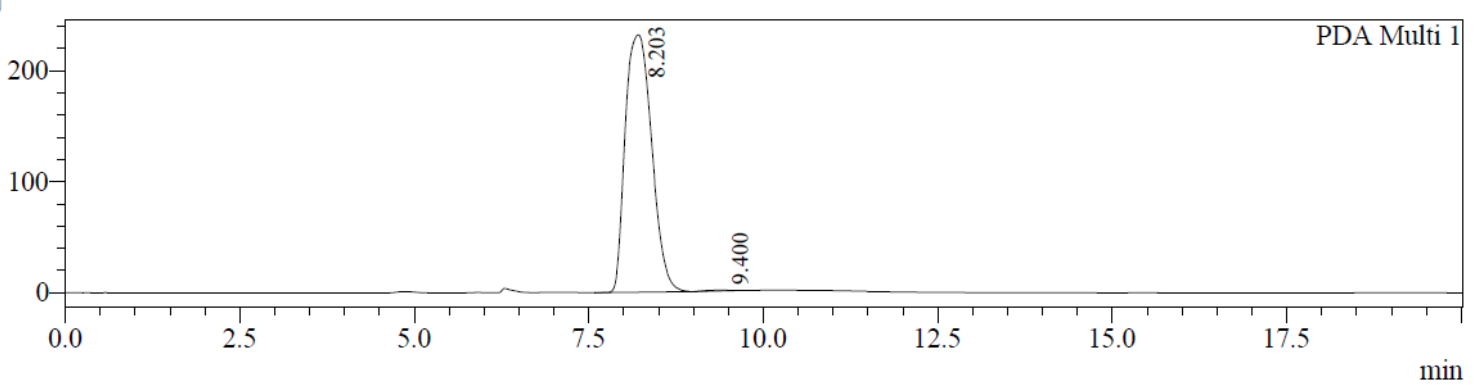

PDA Ch1 254nm 4nm

\begin{tabular}{|r|r|r|r|r|r|}
\hline \multicolumn{1}{|c|}{ Peak\# } & Ret. Time & \multicolumn{1}{|c|}{ Area } & \multicolumn{1}{|c|}{ Height } & \multicolumn{1}{|c|}{ Area \% } & \multicolumn{1}{c|}{ Height \% } \\
\hline 1 & 8.203 & 6137528 & 232089 & 99.636 & 99.634 \\
\hline 2 & 9.400 & 22446 & 853 & 0.364 & 0.366 \\
\hline Total & & 6159974 & 232943 & 100.000 & 100.000 \\
\hline
\end{tabular}

HPLC of pure $\left(\mathrm{S}_{\mathrm{p}}\right)-4: \mathrm{tR}_{1}=8.4 \mathrm{~min} ; \mathrm{tR}_{2}=9.5 \mathrm{~min}, 97 \%$ ee

$\mathrm{mAU}$

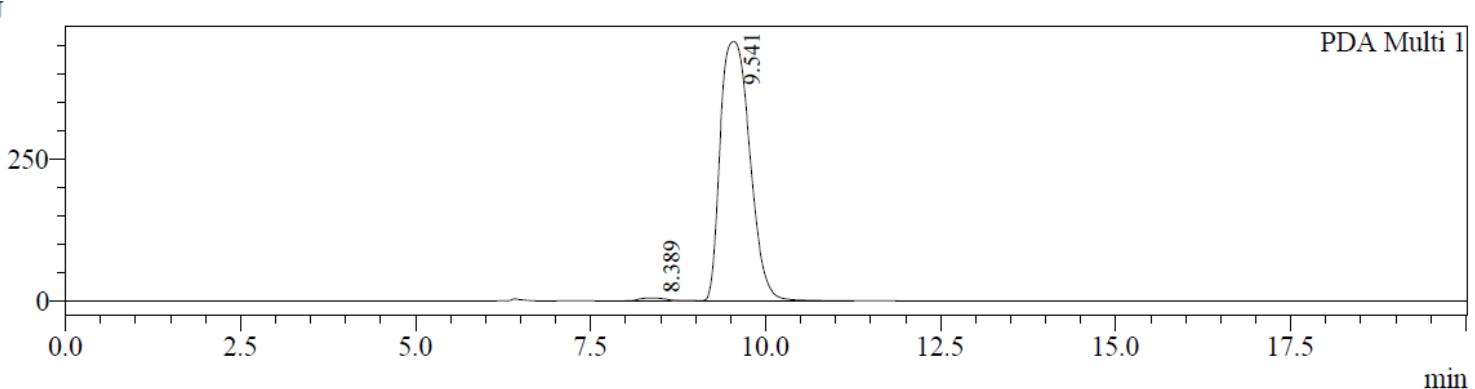

PDA Ch1 254nm 4nm

\begin{tabular}{|r|r|r|r|r|r|}
\hline \multicolumn{1}{|c|}{ Peak\# } & Ret. Time & \multicolumn{1}{|c|}{ Area } & \multicolumn{1}{|c|}{ Height } & \multicolumn{1}{c|}{ Area \% } & \multicolumn{1}{|c|}{ Height \% } \\
\hline 1 & 8.389 & 137533 & 5191 & 1.014 & 1.123 \\
\hline 2 & 9.541 & 13428616 & 456881 & 98.986 & 98.877 \\
\hline Total & & 13566148 & 462072 & 100.000 & 100.000 \\
\hline
\end{tabular}


1-[15-(Dimethylamino)tricyclo[8.2.2.24, 7 hexadeca-1(12),4(16),5,7(15),10,13-hexaen-5yl]propan-1-one 12

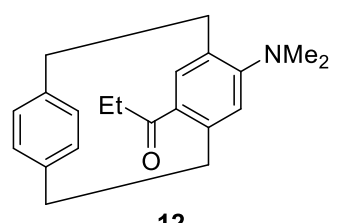

12

mAU
Chiral HPLC analysis : [Column $=$ Chiralcel OJ $(250 \times 4,6 \mathrm{~mm})$; eluent $=n$-heptane $/ i-\operatorname{PrOH}(95: 5) ;$ flow $=1 \mathrm{~mL} / \mathrm{min} ; \lambda=254 \mathrm{~nm}]$

HPLC of racemic compound $( \pm)-12: \mathrm{tR}_{1}=8.5 \mathrm{~min} ; \mathrm{tR}_{2}=12.6 \mathrm{~min}$

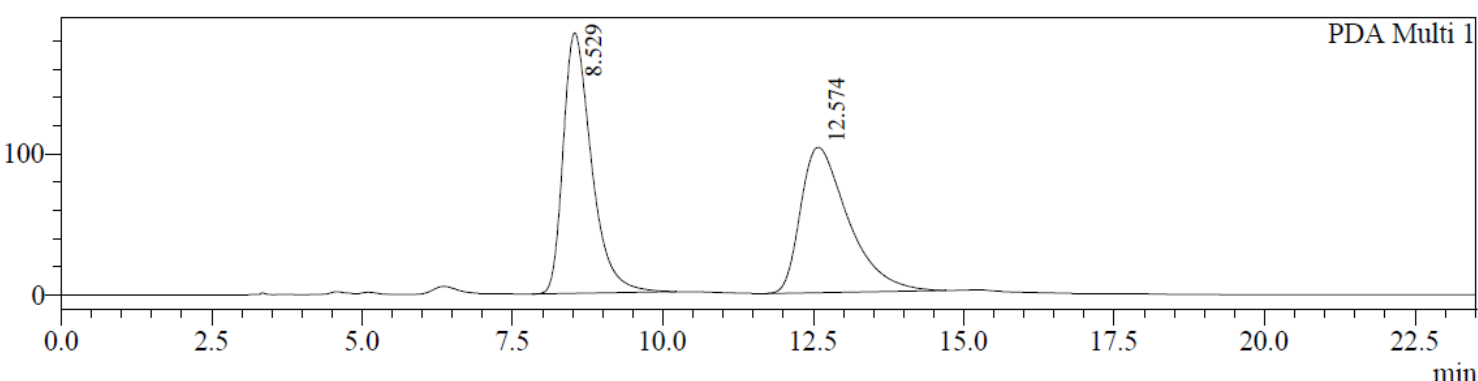

PDA Ch1 $254 \mathrm{~nm} 4 \mathrm{~nm}$
\begin{tabular}{|r|r|r|r|r|r|}
\hline \multicolumn{1}{|c|}{ Peak\# } & Ret. Time & Area & Height & Area \% & Height $\%$ \\
\hline 1 & 8.529 & 6040012 & 184444 & 51.538 & 64.188 \\
\hline 2 & 12.574 & 5679472 & 102904 & 48.462 & 35.812 \\
\hline Total & & 11719484 & 287348 & 100.000 & 100.000 \\
\hline
\end{tabular}

HPLC of pure product $\left(S_{\mathrm{p}}\right)-\mathbf{1 2}: \mathrm{tR}=8.3 \mathrm{~min},>99 \%$ ee

mAU

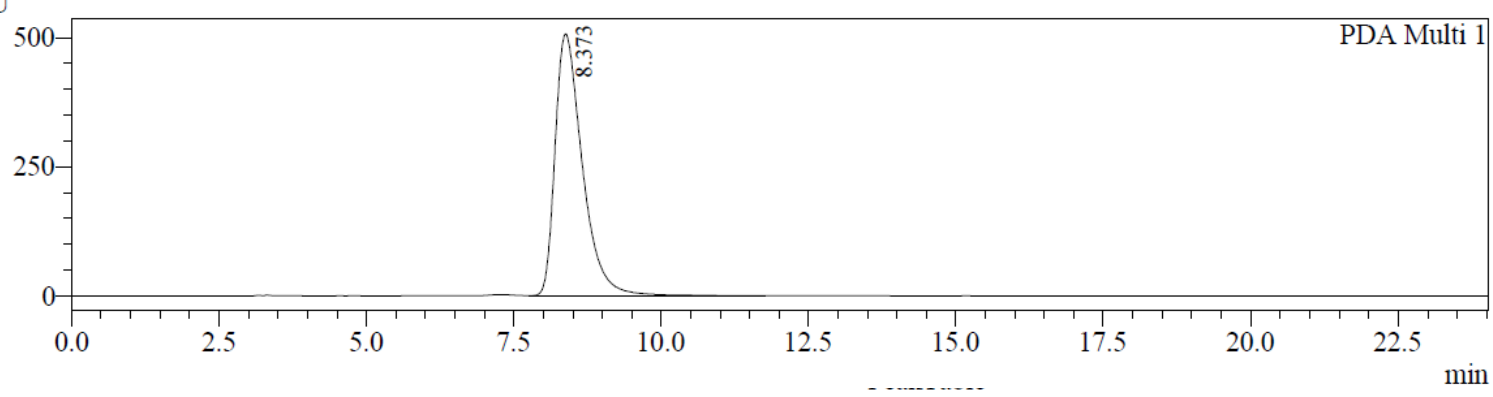

PDA Ch1 254nm 4nm

\begin{tabular}{|r|r|r|r|r|r|}
\hline \multicolumn{1}{|c|}{ Peak\# } & Ret. Time & Area & Height & Area \% & \multicolumn{1}{c|}{ Height \% } \\
\hline 1 & 8.373 & 16332712 & 506661 & 100.000 & 100.000 \\
\hline Total & & 16332712 & 506661 & 100.000 & 100.000 \\
\hline
\end{tabular}

HPLC of pure product $\left(R_{\mathrm{p}}\right)-\mathbf{1 2}: \mathrm{tR}_{1}=8.5 \mathrm{~min} ; \mathrm{tR}_{2}=12.4 \mathrm{~min}, 95 \%$ ee

$\mathrm{mAU}$

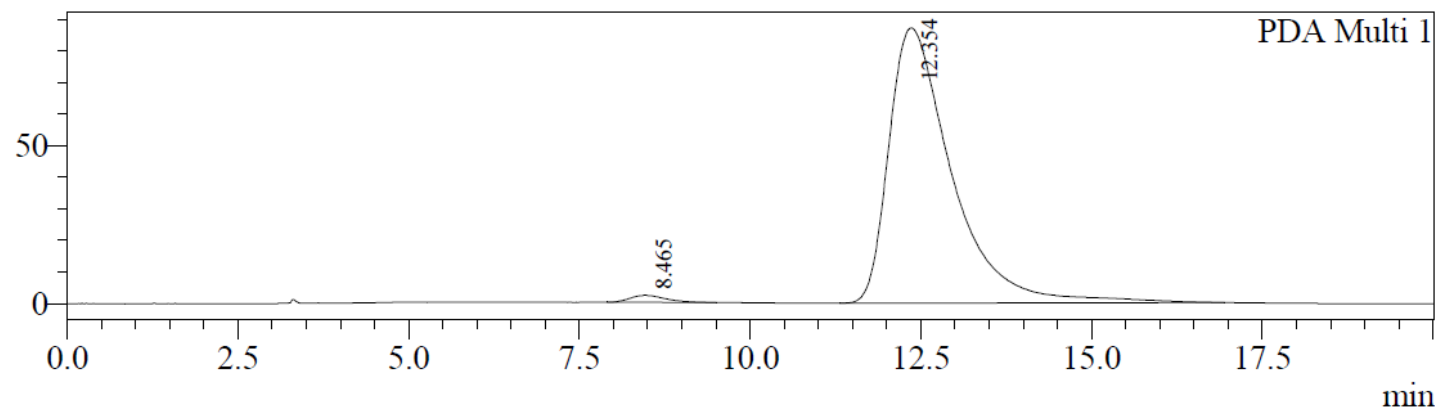

PDA Ch1 254nm 4nm

\begin{tabular}{|r|r|r|r|r|r|}
\hline \multicolumn{1}{|c|}{ Peak\# } & Ret. Time & \multicolumn{1}{c|}{ Area } & Height & \multicolumn{1}{c|}{ Area \% } & Height \% \\
\hline 1 & 8.465 & 81485 & 2224 & 1.432 & 2.488 \\
\hline 2 & 12.354 & 5608144 & 87182 & 98.568 & 97.512 \\
\hline Total & & 5689629 & 89406 & 100.000 & 100.000 \\
\hline
\end{tabular}




\section{Unpolarised spectroscopic studies}

1-[15-(Dimethylamino)tricyclo[8.2.2.24, 7 hexadeca-1(12),4(16),5,7(15),10,13-hexaen-5yl]propan-1-one (( \pm$)-12)$

Photophysical properties in solution
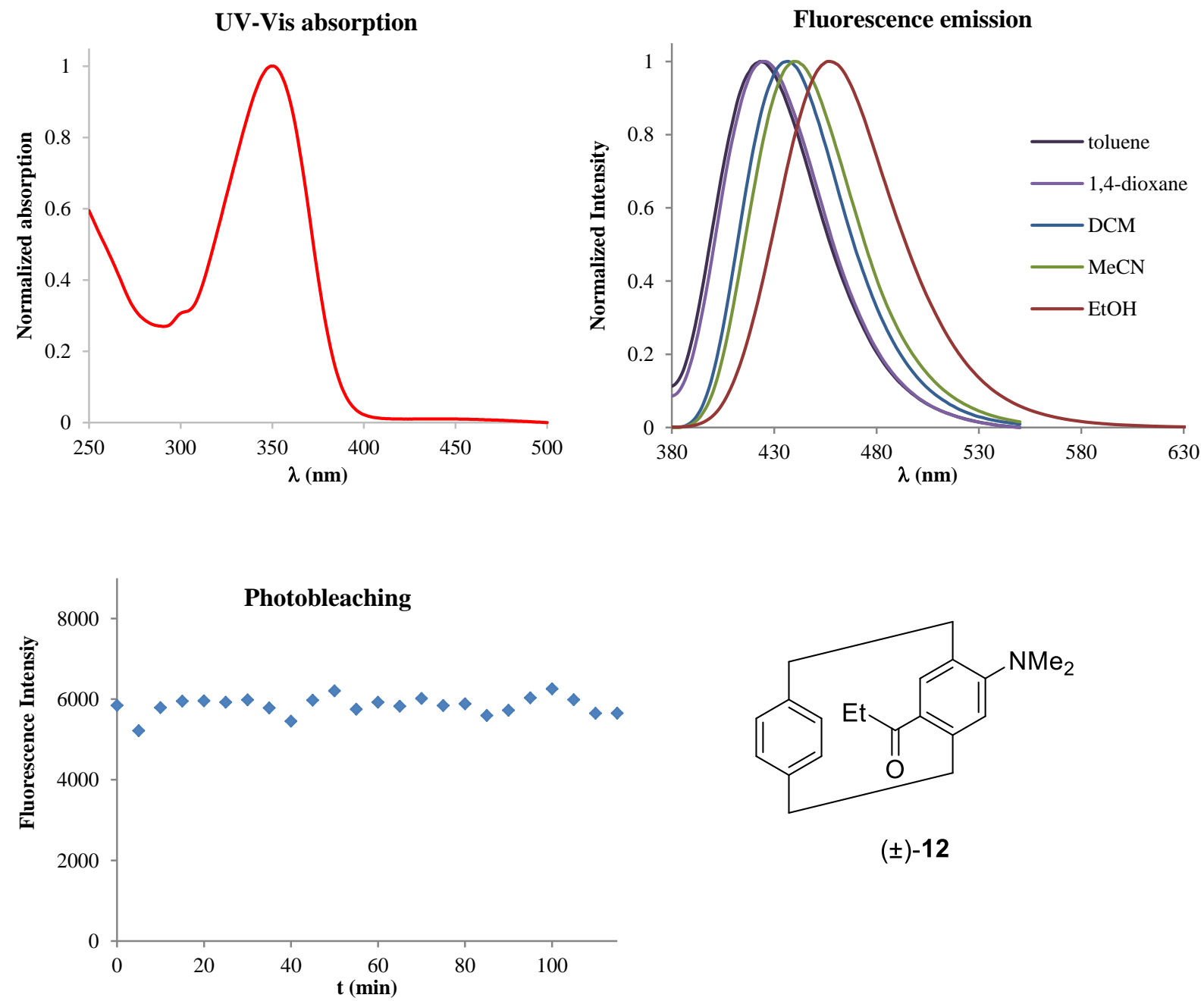

$( \pm)-12$

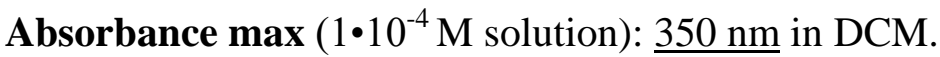

Emission max $\left(1 \cdot 10^{-4} \mathrm{M}\right.$ solutions): $\underline{424 \mathrm{~nm}}$ in toluene $\left(\lambda_{\mathrm{ex}}=350 \mathrm{~nm}\right), \underline{425 \mathrm{~nm}}$ in $1,4-$ dioxane $\left(\lambda_{\mathrm{ex}}=350 \mathrm{~nm}\right), \underline{437 \mathrm{~nm}}$ in $\operatorname{DCM}\left(\lambda_{\mathrm{ex}}=350 \mathrm{~nm}\right) ; \underline{440 \mathrm{~nm}}$ in $\operatorname{MeCN}\left(\lambda_{\mathrm{ex}}=350 \mathrm{~nm}\right)$; $458 \mathrm{~nm}$ in $\mathrm{EtOH}\left(\lambda_{\mathrm{ex}}=350 \mathrm{~nm}\right)$;

QY $\sim 1 \%$ in DCM. Relative quantum yield (QY) was calculated using anthracene in cyclohexane as fluorescence standard $(\mathrm{QY}=36 \%)$. The excitation wavelength was fixed at $340 \mathrm{~nm}$ for both the sample and the standard.

\section{Photobleaching study}

A $1 \cdot 10^{-4} \mathrm{M}$ solution of product $( \pm)-\mathbf{1 2}$ in DCM was used to perform the photobleaching study. The sample was continuously irradiated for $115 \mathrm{~min}$ at $20{ }^{\circ} \mathrm{C}\left(\lambda_{\mathrm{ex}}=350 \mathrm{~nm}\right)$ and maximum emission intensity changes $\left(\lambda_{\max }=437 \mathrm{~nm}\right)$ were measured at 5 -minute time intervals. 
1-[14-(Dimethylamino)-tricyclo[8.2.2.2^\{4,7\}]hexadeca-1(12),4,6,10,13,15-hexaen-5-yl]propan-1-one (( \pm$)-4)$

\section{Photophysical properties in solution}
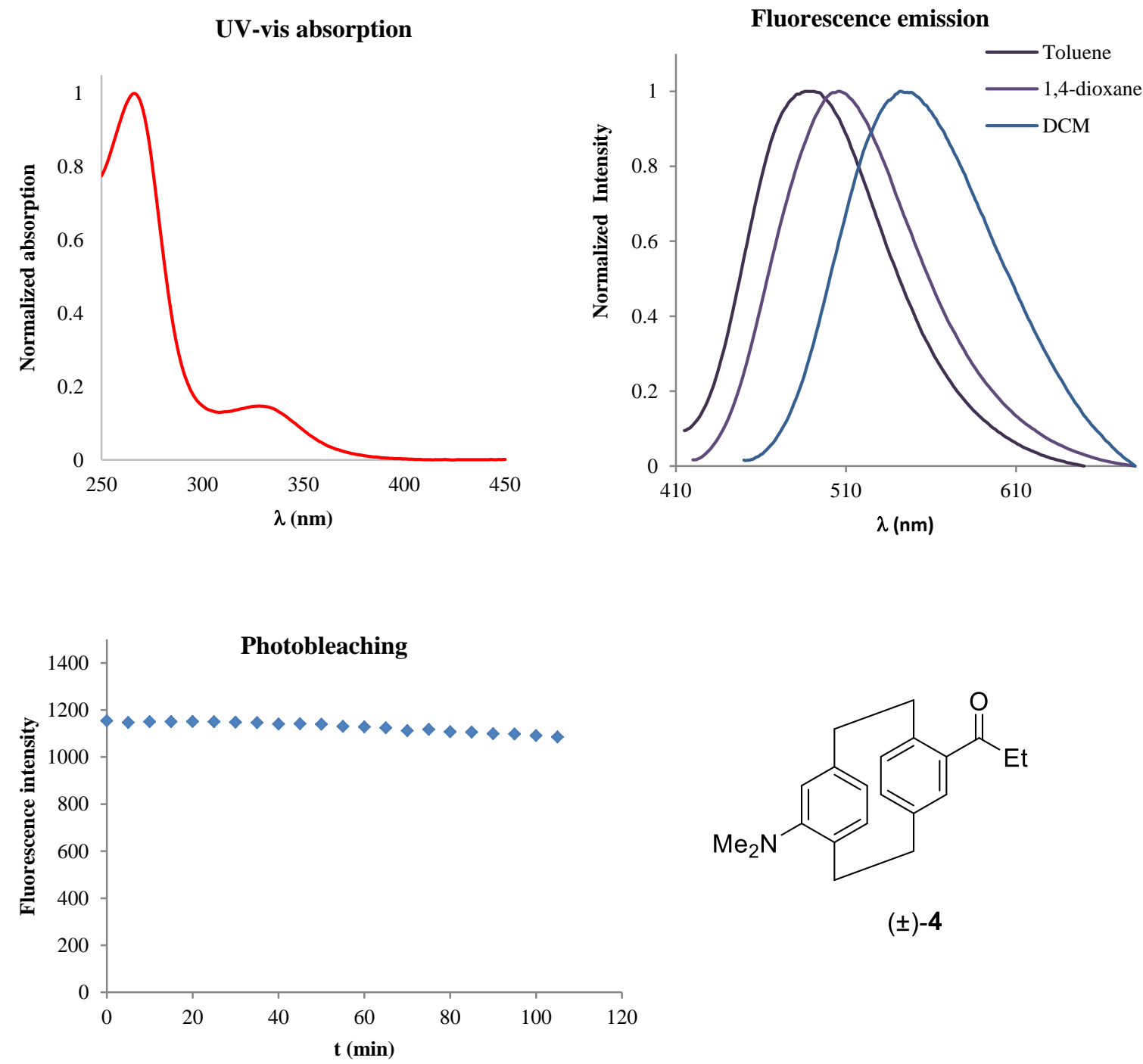

$( \pm)-4$

Absorbance max $\left(1 \cdot 10^{-4} \mathrm{M}\right.$ solution): $\underline{266}$ and $\underline{328 \mathrm{~nm}}$ in DCM.

Emission max $\left(1 \cdot 10^{-4} \mathrm{M}\right.$ solutions): $\underline{486 \mathrm{~nm}}$ in toluene $\left(\lambda_{\mathrm{ex}}=350 \mathrm{~nm}\right), \underline{506 \mathrm{~nm}}$ in 1,4dioxane $\left(\lambda_{\mathrm{ex}}=350 \mathrm{~nm}\right), \underline{542 \mathrm{~nm}}$ in DCM $\left(\lambda_{\mathrm{ex}}=350 \mathrm{~nm}\right)$.

QY. $<0.1 \%$. Relative quantum yield (QY) was calculated using anthracene in cyclohexane as fluorescence standard $(\mathrm{QY}=36 \%)$. The excitation wavelength was fixed at $340 \mathrm{~nm}$ for both the sample and the standard.

\section{Photobleaching study}

A $1 \cdot 10^{-4} \mathrm{M}$ solution of product ( \pm )-4 in DCM was used to perform the photobleaching study. The sample was continuously irradiated for $105 \mathrm{~min}$ at $20^{\circ} \mathrm{C}\left(\lambda_{\mathrm{ex}}=350 \mathrm{~nm}\right)$ and maximum emission intensity changes $\left(\lambda_{\max }=545 \mathrm{~nm}\right)$ were measured at 5-minute time intervals. 
Excitation-emission map at the solid state

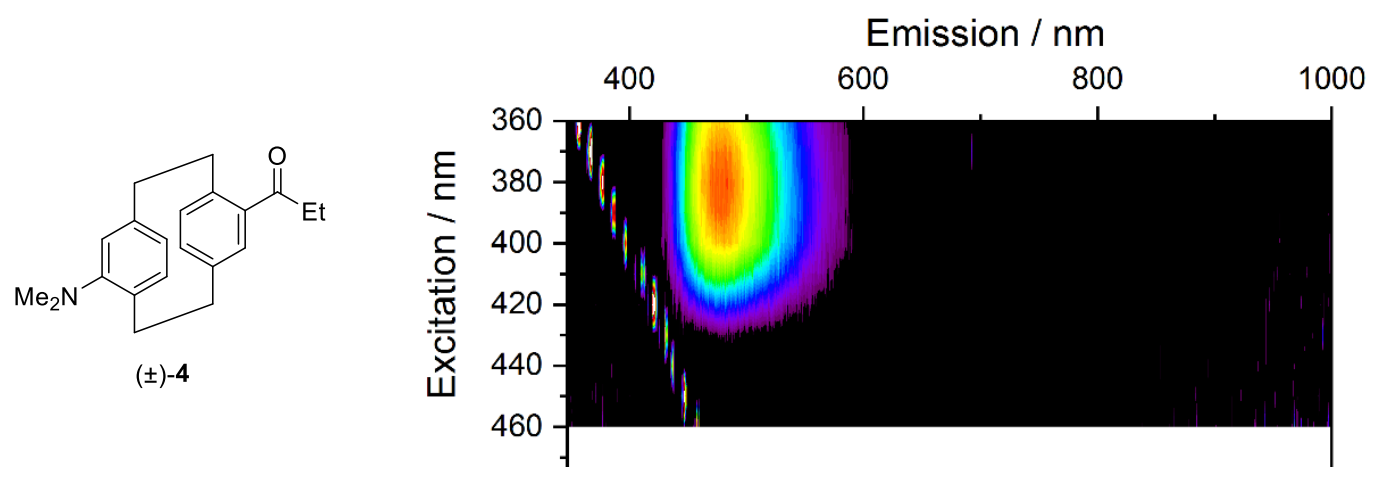




\section{Theoretical investigations}

Cartesian coordinates can be found in text format in another supporting file (archive).

Benchmark on the photophysical properties of 2D-PRODAN. Focusing on the main photophysical properties of the 2D PRODAN, three functionals are tested and their ability to reproduce both the solvatochromic and solvato-fluorochromic shift are evaluated. The PBE0 functional (with $\mathrm{D} 3^{\mathrm{BJ}}$ dispersion corrections) is dismissed due to (i) the large red-shift in the absorption energies and (ii) the largest errors on the $\mathrm{E}_{00}$ values. M06-2X shows an inversion of the first two excited states between gas and condensed phase. $\omega \mathrm{B} 97 \mathrm{X}-\mathrm{D}$ functional is selected as it yields to the closest theoretical solvatochromic shift between Tol and ACN compared to the experimental one. A second argument being that $\omega \mathrm{B} 97-\mathrm{XD}$ is a range-separated functional well-suited to accurately describe charge-transfer transitions, which should be essential in our case (see Table S2)

Table S1 : Benchmark on transitions for 2D-PRODAN. The GS geometries were computed using the 6-31+G(d) basis-set using the same functionals, TD-DFT computations with the 6-311+G(2d,p) basis-set. Energies are given in eV, wavelength in nm. ${ }^{a}$ Vertical transitions energies of $\mathrm{S}_{0} \rightarrow \mathrm{S}_{1}$ (abs) or $\mathrm{S}_{1} \rightarrow \mathrm{S}_{0}$ (em). ${ }^{b}$ With empirical dispersion GD3BJ model. ${ }^{c}$ Considering $\mathrm{S}_{0} \rightarrow \mathrm{S}_{1}$ in solvent (PCM) and $\mathrm{S}_{0} \rightarrow \mathrm{S}_{2}$ in gas phase. ${ }^{d} \mathrm{RMS}$ calculated on the three solvent for the same functional.

\begin{tabular}{|c|c|c|c|c|c|c|c|c|c|}
\hline Functional & Solvent & $\Delta \mathbf{E}_{\boldsymbol{a b s}}^{\boldsymbol{t h}{ }^{a}}$ & $\lambda_{a b s}^{t h}$ & $\lambda_{a b s}^{e x p t}$ & $\Delta \mathbf{E}_{\text {em }}^{\text {th }}{ }^{a}$ & $\lambda_{e m}^{t h}$ & $\lambda_{e m}^{e x p t}$ & $\mathrm{E}_{0-0}^{t h}$ & $\mathbf{E}_{0-0}^{\text {expt }}$ \\
\hline \multirow[t]{4}{*}{$\mathrm{PBE} \mathrm{PBE}^{b}$} & $\mathrm{ACN}$ & 3.224 & 385 & 350 & 2.691 & 461 & 455 & 2.835 & 3.134 \\
\hline & DCM & 3.239 & 383 & 355 & 2.802 & 442 & 440 & 2.920 & 3.155 \\
\hline & Tol & 3.332 & 372 & 346 & 3.062 & 405 & 405 & 3.123 & 3.282 \\
\hline & $\Delta \mathrm{E}^{A C N-T o l}$ & \multicolumn{2}{|c|}{0.109} & 0.043 & \multicolumn{2}{|c|}{0.370} & 0.255 & 0.288 & 0.148 \\
\hline \multirow[t]{4}{*}{ M06-2X } & $\mathrm{ACN}$ & 3.348 & 370 & 350 & 2.949 & 420 & 450 & 3.155 & 3.134 \\
\hline & DCM & 3.428 & 362 & 355 & 3.059 & 405 & 440 & 3.236 & 3.155 \\
\hline & Tol & 3.682 & 337 & 346 & 3.314 & 374 & 405 & 3.430 & 3.282 \\
\hline & $\Delta \mathrm{E}^{A C N-T o l}$ & \multicolumn{2}{|c|}{0.335} & 0.043 & \multicolumn{2}{|c|}{0.365} & 0.255 & 0.275 & 0.148 \\
\hline \multirow[t]{4}{*}{$\omega \mathrm{B} 97-\mathrm{XD}$} & $\mathrm{ACN}$ & 3.694 & 355 & 350 & 3.002 & 413 & 450 & 3.211 & 3.134 \\
\hline & DCM & 3.705 & 347 & 355 & 3.102 & 399 & 440 & 3.285 & 3.155 \\
\hline & Tol & 3.711 & 329 & 346 & 3.340 & 371 & 405 & 3.463 & 3.282 \\
\hline & $\Delta \mathrm{E}^{A C N-T o l}$ & \multicolumn{2}{|c|}{0.077} & 0.043 & \multicolumn{2}{|c|}{0.339} & 0.255 & 0.252 & 0.148 \\
\hline
\end{tabular}

Table S2: $\mathrm{PCM}($ Toluene ) - $\omega \mathrm{B} 97 \mathrm{X}-\mathrm{D} / 6-311+\mathrm{G}(2 \mathrm{~d}, \mathrm{p}) / / \omega \mathrm{B} 97 \mathrm{X}-\mathrm{D} / 6-31+\mathrm{G}(\mathrm{d})$ Vertical transitions characteristics of 2DPRODAN in Toluene

Absorption

Emission

\begin{tabular}{|c|c|c|c|c|c|c|c|c|c|c|c|}
\hline $\mathrm{ES}$ & $\Delta \mathrm{E}_{a b s}^{t h}$ & $\mathrm{f}$ & $\lambda_{a b s}^{t h}$ & $\Delta \mu(\mathrm{D})$ & $\mathrm{D}(\AA)$ & $\mathrm{QP}(\mathrm{au})$ & MO contrib. & $\mathrm{ES}$ & $\Delta \mathrm{E}_{e m}^{t h}$ & $\mathrm{f}$ & $\lambda_{e m}^{t h}$ \\
\hline $\mathrm{S}_{1}$ & 3.711 & 0.40 & 329 & 5.79 & 2.294 & 0.535 & $80 \% \mathrm{H} \rightarrow \mathrm{L}$ & $\mathrm{S}_{1}$ & 3.340 & 0.47 & 371 \\
\hline
\end{tabular}




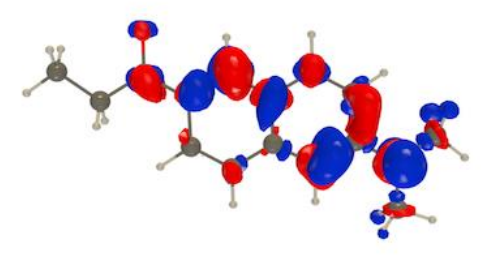

EDD $S_{1}$

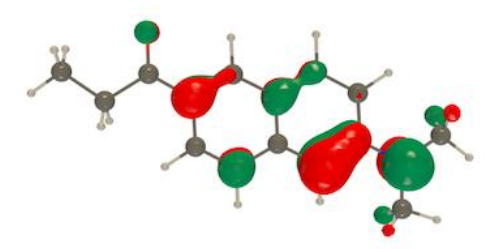

HOMO (61)

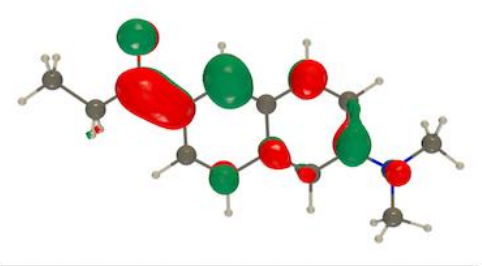

LUMO (63)

Figure S1: PCM (Toluene) - $\omega \mathrm{B} 97 \mathrm{X}-\mathrm{D} / 6-311+\mathrm{G}(2 \mathrm{~d}, \mathrm{p}) / / \omega \mathrm{B} 97 \mathrm{X}-\mathrm{D} / 6-31+\mathrm{G}(\mathrm{d})$ HOMO, LUMO and EDD plots for 2DPRODAN. MO isovalue: 0.05 au \& EDD isovalue: 0.002 au. The red and blue lobes of the EDD indicate increase and decrease of the electron density upon absorption, respectively.

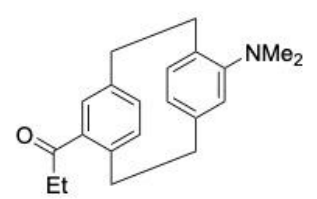

$4(79 \%)$

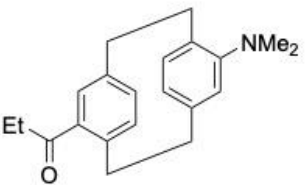

$4 \mathrm{~b}(21 \%)$

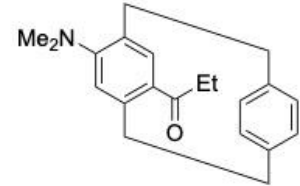

$12(89 \%)$

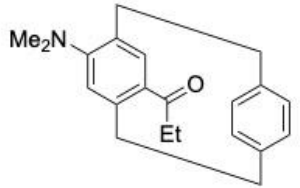

$12 \mathrm{~b}(11 \%)$

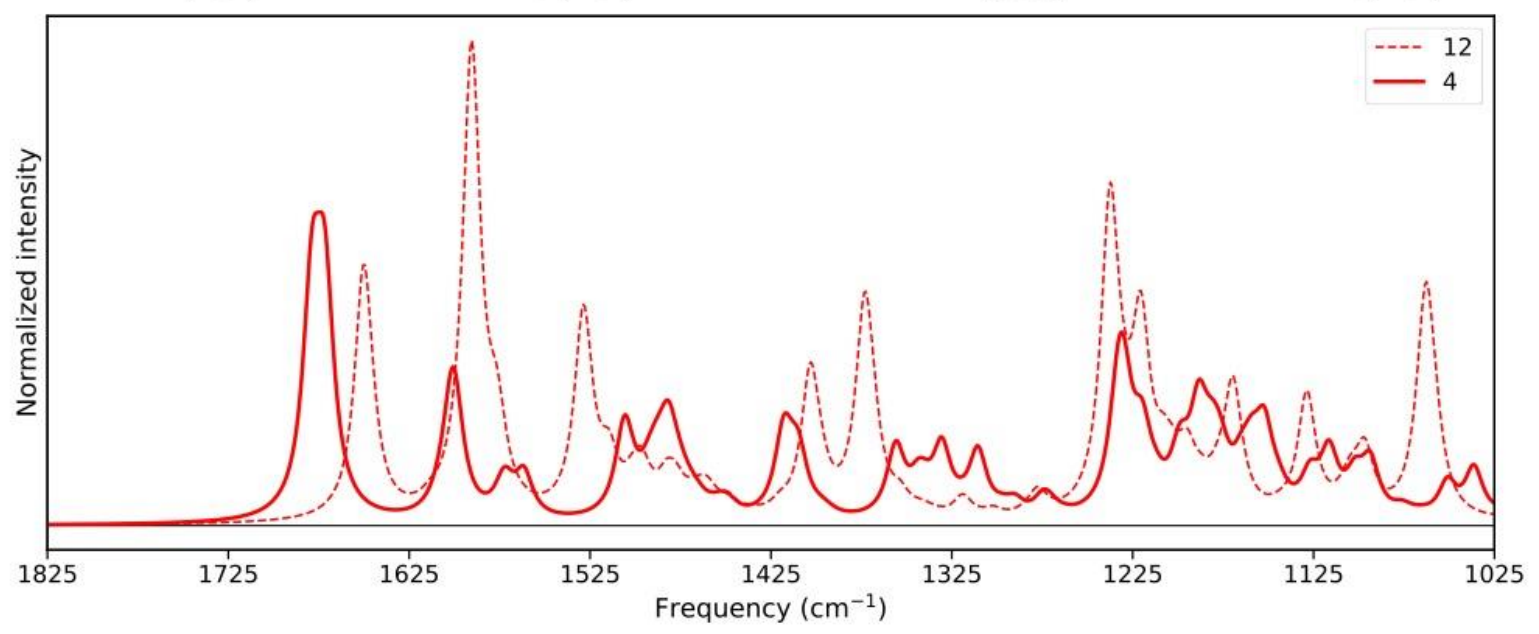

Figure S2: PCM(DCM) - B3LYP/6-31+G(d) simulated IR for compound $\mathbf{4}$ and $\mathbf{1 2}$ in DCM. Frequencies have been scaled by 0.98 . Sticks have been broadened by Lorentzian functions with FWHM $=6 \mathrm{~cm}^{-1}$. Each spectrum is weighted by the Boltzmann population of the two conformers at $298 \mathrm{~K}$. 


\section{Theoretical study on compound 12:}

Table S3: PCM(1,4-dioxane) - $\omega \mathrm{B} 97 \mathrm{X}-\mathrm{D} / 6-311+\mathrm{G}(2 \mathrm{~d}, \mathrm{p}) / / \omega \mathrm{B} 97 \mathrm{X}-\mathrm{D} / 6-31+\mathrm{G}(\mathrm{d})$. Principal excited states characteristics of 12 in 1,4-dioxane- Transition energies are calculated using Eq. 1

\begin{tabular}{|c|c|c|c|c|c|c|c|c|c|c|c|}
\hline $\mathbf{E S}$ & $\mathbf{\%}$ & $\mathbf{M O}$ & $\Delta \mathbf{E}_{\boldsymbol{a b s}}^{\boldsymbol{t h}}$ & $\mathbf{f}$ & $\lambda_{\boldsymbol{a b s}}^{\boldsymbol{t h}}$ & $\Delta \boldsymbol{\mu}(\mathbf{D})$ & $\mathbf{D}(\AA)$ & $\mathbf{Q P}(\mathbf{a u})$ & $\Delta \mathbf{E}_{\boldsymbol{e m}}^{\boldsymbol{t h}}$ & $\mathbf{f}$ & $\lambda_{\boldsymbol{e m}}^{\text {th }}$ \\
\hline 1 & $\begin{array}{c}45 \% \\
32 \%\end{array}$ & $\begin{array}{c}\mathrm{H} \rightarrow \mathrm{L} \\
\mathrm{H}-3 \rightarrow \mathrm{L}\end{array}$ & 4.029 & 0.159 & 308 & 0.22 & 0.122 & 0.590 & $/$ & $/$ & $/$ \\
\hline 2 & $\begin{array}{c}48 \% \\
25 \%\end{array}$ & $\begin{array}{c}\mathrm{H} \rightarrow \mathrm{L} \\
\mathrm{H}-3 \rightarrow \mathrm{L}\end{array}$ & 4.111 & 0.197 & 302 & 0.86 & 0.375 & 0.576 & $/$ & $/$ & $/$ \\
\hline 3 & $\begin{array}{c}36 \% \\
25 \%\end{array}$ & $\begin{array}{c}\mathrm{H} \rightarrow \mathrm{L}+1 \\
\mathrm{H}-1 \rightarrow \mathrm{L}+1\end{array}$ & 4.239 & 0.002 & 293 & 1.31 & 0.903 & 0.343 & & & \\
\hline
\end{tabular}

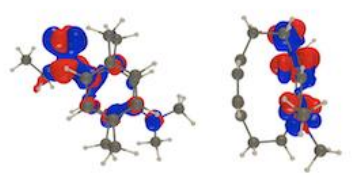

EDD $S_{1}$

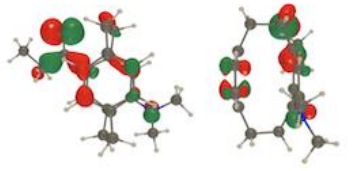

HOMO-3 (80)

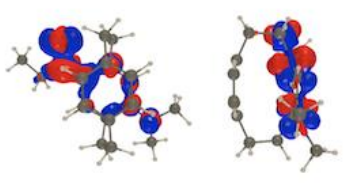

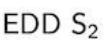

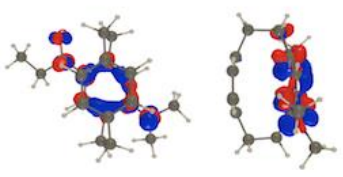

EDD $S_{3}$

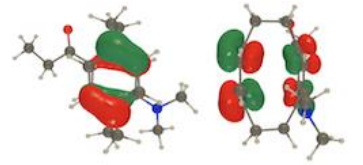

HOMO-1 (82)

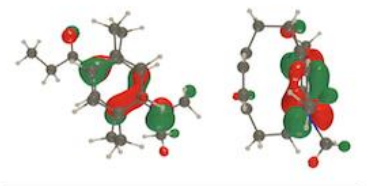

HOMO (83)

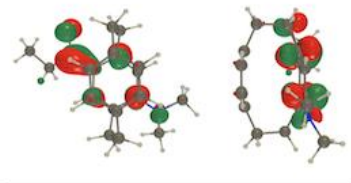

LUMO (84)

Figure S3: PCM (1,4-dioxane) - $\omega$ B97X-D/ 6-311+G(2d,p) // $\omega$ B97X-D/6-31+G(d). HOMO, LUMO and EDD plots for compound 12 in 1,4-dioxane. MO isovalue: 0.05 au \& EDD isovalue: 0.002 au. The red and blue lobes of the EDD indicate increase and decrease of the electron density upon absorption, respectively.

Table S4: PCM(Toluene) - $\omega$ B97X-D/ 6-311+G(2d,p) // $\omega$ B97X-D/6-31+G(d). Principal excited states characteristics of 12 in Toluene- Transition energies are calculated using Eq. 1

\begin{tabular}{|c|c|c|c|c|c|c|c|c|c|c|c|}
\hline $\mathbf{E S}$ & $\mathbf{\%}$ & $\mathbf{M O}$ & $\Delta \mathbf{E}_{\boldsymbol{a b s}}^{\boldsymbol{t h}}$ & $\mathbf{f}$ & $\lambda_{\boldsymbol{a b s}}^{\text {th }}$ & $\Delta \boldsymbol{\mu}(\mathbf{D})$ & $\mathbf{D}(\mathbf{\AA})$ & $\mathbf{Q P}(\mathbf{a u})$ & $\Delta \mathbf{E}_{\boldsymbol{e m}}^{\boldsymbol{t h}}$ & $\mathbf{f}$ & $\boldsymbol{\lambda}_{\boldsymbol{e m}}^{\text {th }}$ \\
\hline 1 & $\begin{array}{c}53 \% \\
27 \%\end{array}$ & $\begin{array}{c}\mathrm{H} \rightarrow \mathrm{L} \\
\mathrm{H}-4 \rightarrow \mathrm{L}\end{array}$ & 4.023 & 0.197 & 308 & 3.84 & 1.529 & 0.557 & $/$ & $/$ & $/$ \\
\hline 2 & $\begin{array}{c}40 \% \\
30 \%\end{array}$ & $\begin{array}{c}\mathrm{H} \rightarrow \mathrm{L} \\
\mathrm{H}-3 \rightarrow \mathrm{L}\end{array}$ & 4.105 & 0.173 & 302 & -1.99 & 0.657 & 0.665 & $/$ & $/$ & $/$ \\
\hline 3 & $\begin{array}{c}36 \% \\
25 \%\end{array}$ & $\begin{array}{c}\mathrm{H} \rightarrow \mathrm{L}+1 \\
\mathrm{H}-1 \rightarrow \mathrm{L}+1\end{array}$ & 4.236 & 0.002 & 293 & 1.43 & 0.927 & 0.359 & & & \\
\hline
\end{tabular}

Table S5: PCM(DCM) - $\omega$ B97X-D/ 6-311+G(2d,p) // $\omega$ B97X-D/6-31+G(d) Principal excited states characteristics of 12 in DCM- Transition energies are calculated using Eq. 1

\begin{tabular}{|c|c|c|c|c|c|c|c|c|c|c|c|}
\hline $\mathbf{E S}$ & $\mathbf{\%}$ & $\mathbf{M O}$ & $\Delta \mathbf{E}_{\boldsymbol{a} \boldsymbol{b s}}^{\boldsymbol{t h}}$ & $\mathbf{f}$ & $\lambda_{\boldsymbol{a b s}}^{\boldsymbol{t h}}$ & $\Delta \boldsymbol{\mu}(\mathbf{D})$ & $\mathbf{D}(\mathbf{\AA})$ & $\mathbf{Q P}(\mathbf{a u})$ & $\Delta \mathbf{E}_{\boldsymbol{e m}}^{\boldsymbol{t h}}$ & $\mathbf{f}$ & $\lambda_{\boldsymbol{e m}}^{\text {th }}$ \\
\hline 1 & $93 \%$ & $\mathrm{H} \rightarrow \mathrm{L}$ & 3.912 & 0.366 & 317 & 4.92 & 1.888 & 0.562 & 3.189 & 0.431 & 389 \\
\hline 2 & $\begin{array}{c}47 \% \\
33 \%\end{array}$ & $\begin{array}{c}\mathrm{H}-3 \rightarrow \mathrm{L} \\
\mathrm{H}-4 \rightarrow \mathrm{L}\end{array}$ & 4.046 & 0.008 & 306 & -3.47 & 1.012 & 0.775 & $/$ & $/$ & $/$ \\
\hline 3 & $\begin{array}{c}35 \% \\
28 \%\end{array}$ & $\begin{array}{c}\mathrm{H} \rightarrow \mathrm{L}+1 \\
\mathrm{H}-1 \rightarrow \mathrm{L}+1\end{array}$ & 4.188 & 0.004 & 296 & & & & & & \\
\hline
\end{tabular}




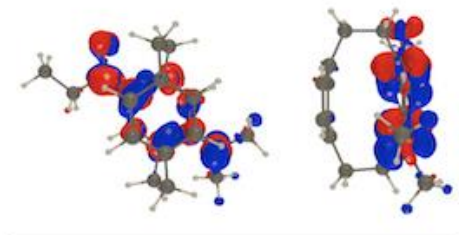

EDD $_{1}$

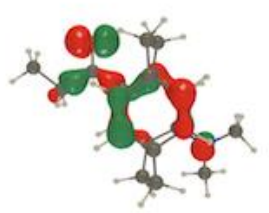

HOMO-4 (79)
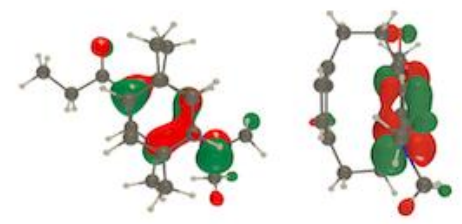

HOMO (83)

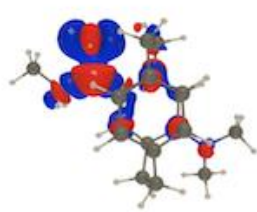

$\mathrm{EDD} \mathrm{S}_{2}$
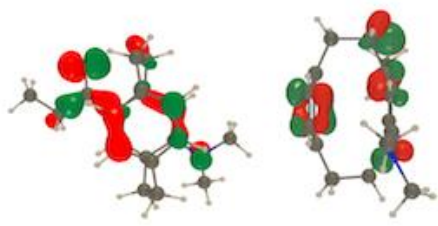

HOMO-3 (80)

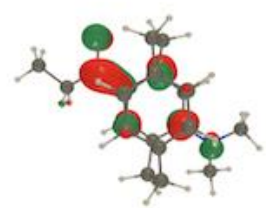

LUMO (84)

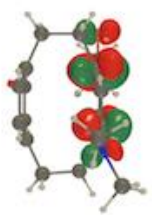

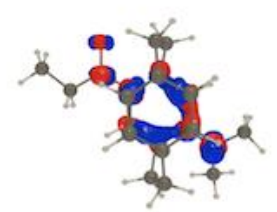

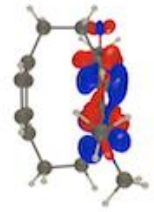

$\mathrm{EDD} \mathrm{S}_{3}$

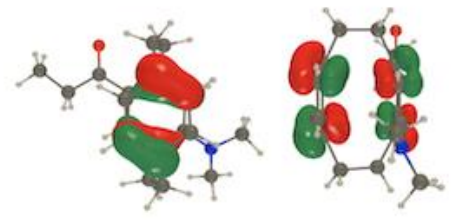

HOMO-1 (82)
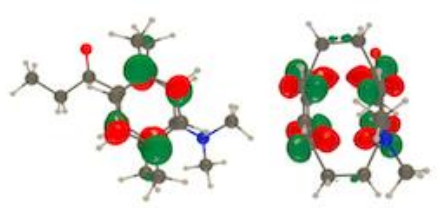

LUMO+1 (85)

Figure S4: PCM (DCM) - $\omega$ B97X-D/ 6-311+G(2d,p) // $\omega B$ 97X-D/6-31+G(d). HOMO, LUMO and EDD plots for compound 12 in DCM. MO isovalue: 0.05 au \& EDD isovalue: 0.002 au. The red and blue lobes of the EDD indicate increase and decrease of the electron density upon absorption, respectively. 


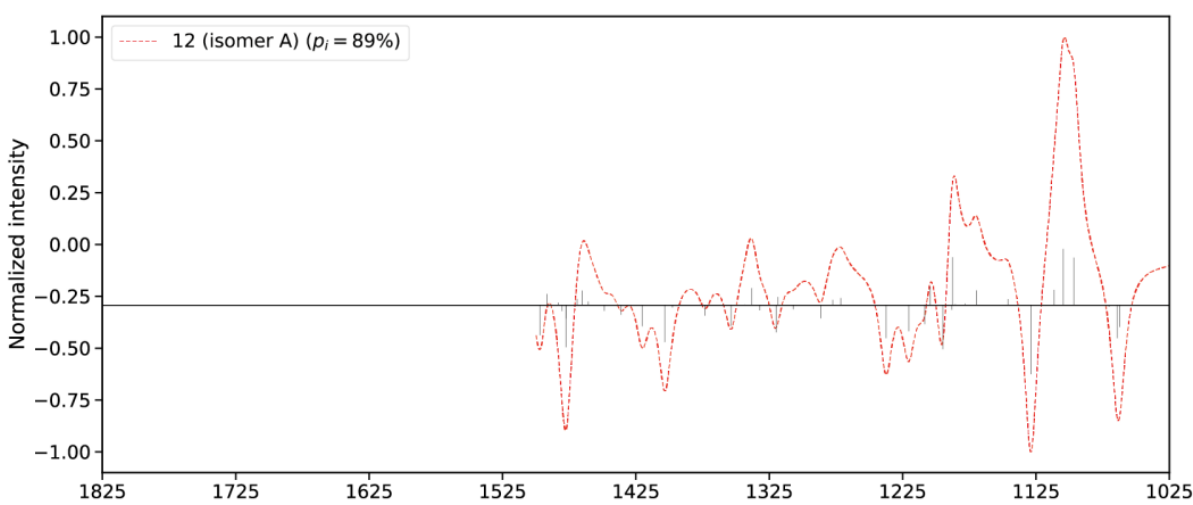

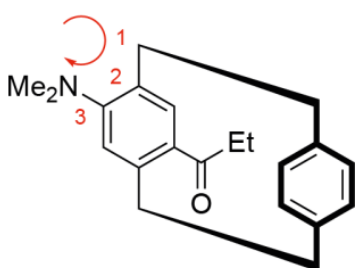

$\left(R_{\mathrm{p}}\right)-12$

$\mathrm{p}_{\mathrm{i}}=89 \%$
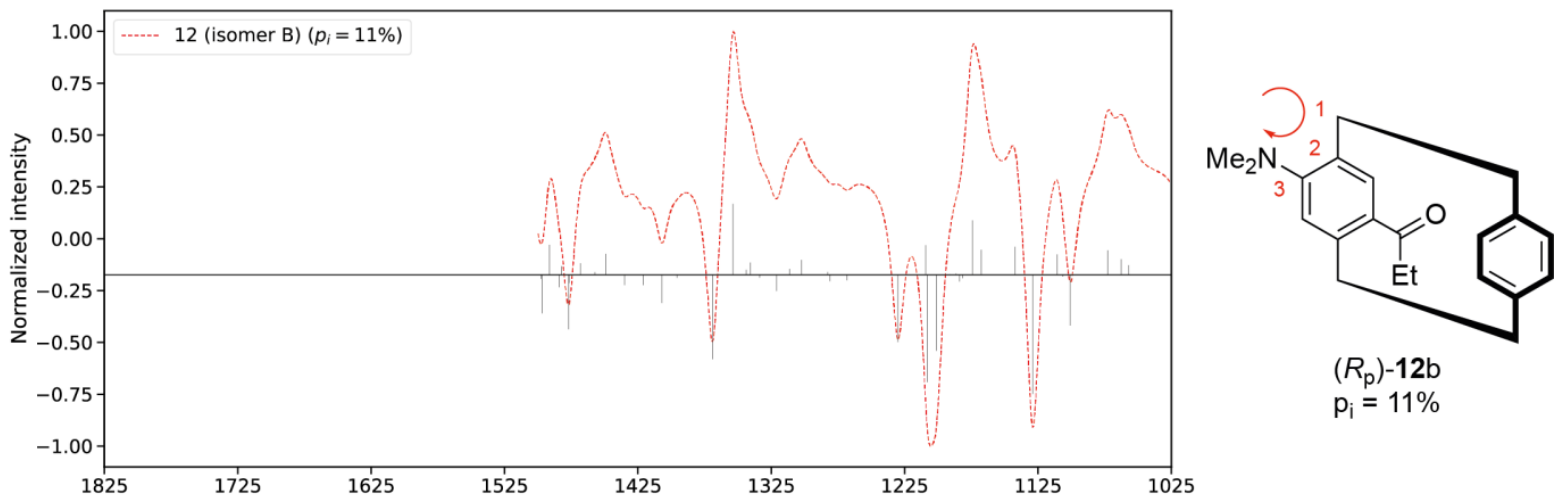

$\left(R_{\mathrm{p}}\right)-12 \mathrm{~b}$

$\mathrm{p}_{\mathrm{i}}=11 \%$

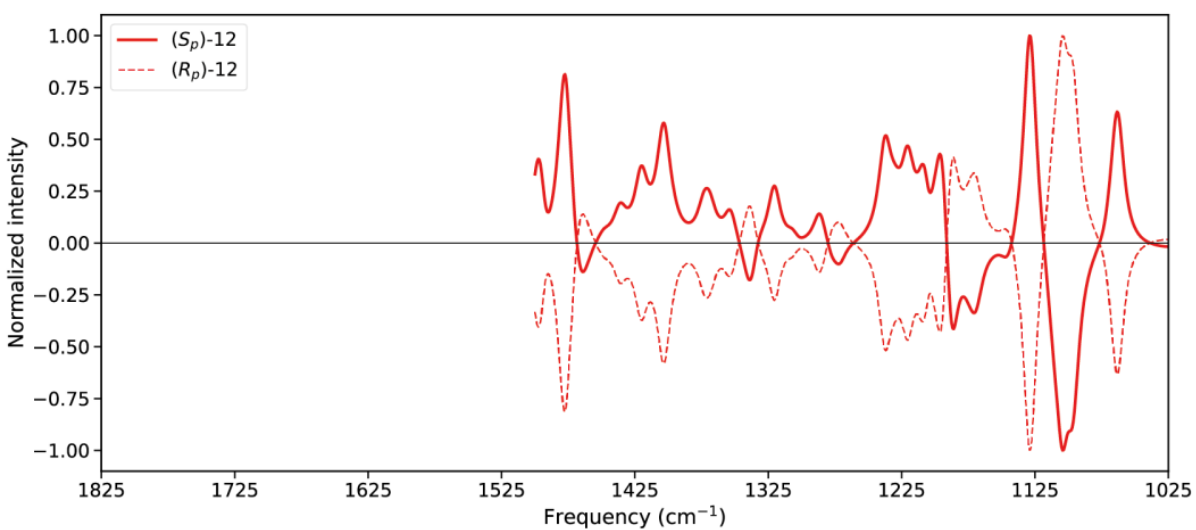

Figure S5: PCM(DCM) - B3LYP/6-31+G(d) simulated VCD for compound 12 in DCM. Frequencies have been scaled by 0.98. Sticks have been broadened by Lorentzian functions with FWHM $=6 \mathrm{~cm}^{-1}$. $\left(\mathrm{R}_{\mathrm{p}}\right)-\mathbf{1 2}$ spectrum on the bottom is weighted by the Boltzmann population of the two conformers at $298 \mathrm{~K}$ displayed at the top, $\left(\mathrm{S}_{\mathrm{p}}\right)-\mathbf{1 2}$ spectrum is obtained mirroring $\left(\mathrm{R}_{\mathrm{p}}\right) \mathbf{- 1 2}$. 

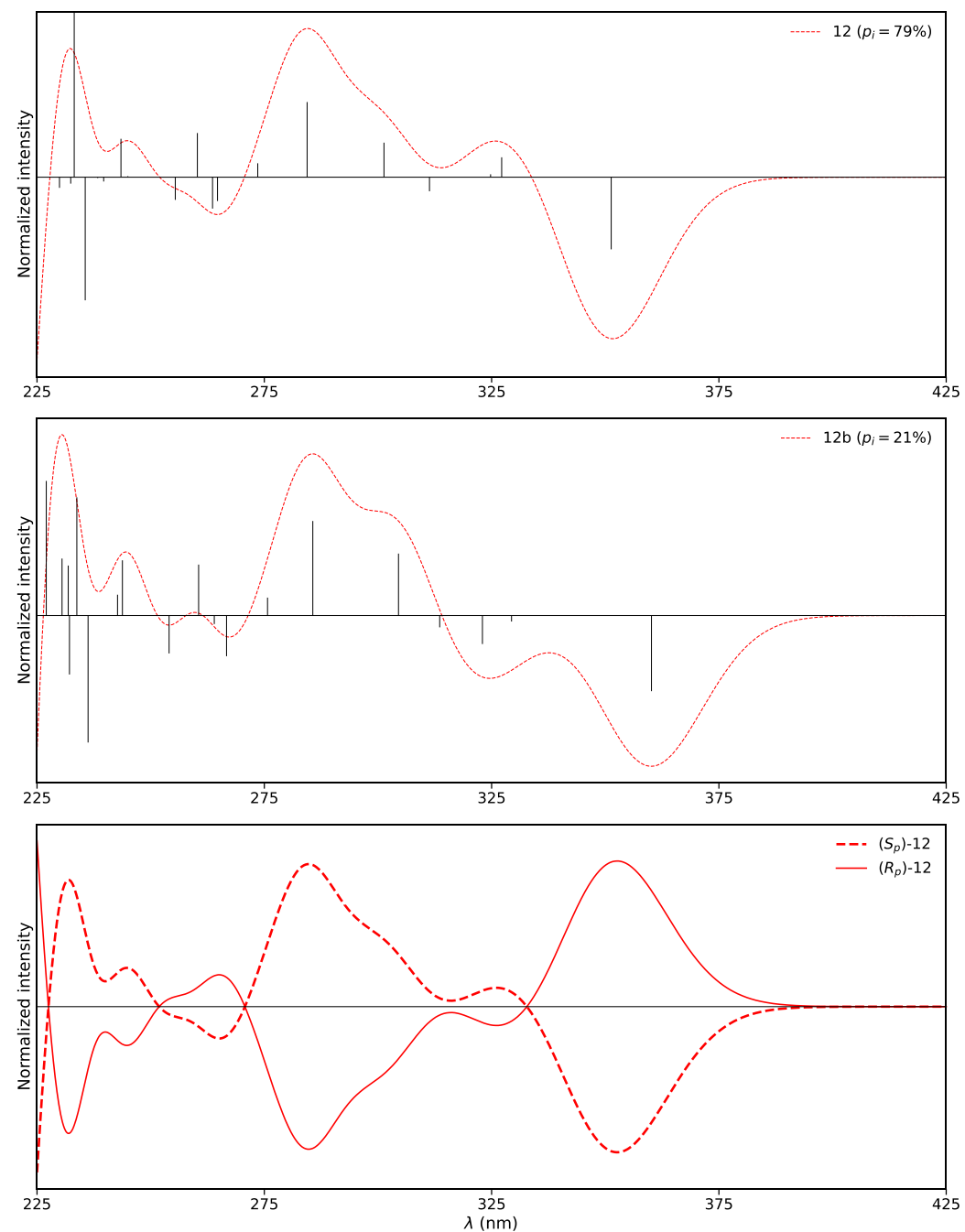

Figure S6: PCM(DCM) - B3LYP/6-311+G(2d,p) simulated ECD for compound 12 in DCM. Sticks have been broadened by Gaussian functions with FWHM $=0.15 \mathrm{eV}$. $\left(\mathrm{R}_{\mathrm{p}}\right)-\mathbf{1 2}$ spectrum on the bottom is weighted by the Boltzmann population of the two conformers (vide supra) at $298 \mathrm{~K},\left(\mathrm{~S}_{\mathrm{p}}\right)-\mathbf{1 2}$ spectrum is obtained mirroring $\left(\mathrm{R}_{\mathrm{p}}\right)-\mathbf{1 2}$. 


\section{Theoretical study on compound 4:}

Table S6: PCM(1,4-dioxane) - $\omega$ B97X-D/ 6-311+G(2d,p) // $\omega$ B97X-D/6-31+G(d). Principal excited states characteristics of 4 in 1,4-dioxane - Transition energies are calculated using Eq. 1

\begin{tabular}{|c|c|c|c|c|c|c|c|c|c|c|c|}
\hline $\mathbf{E S}$ & $\mathbf{\%}$ & $\mathbf{M O}$ & $\Delta \mathbf{E}_{\boldsymbol{a b s}}^{\boldsymbol{t h}}$ & $\mathbf{f}$ & $\lambda_{\boldsymbol{a b s} \boldsymbol{s}}^{\boldsymbol{t h}}$ & $\Delta \boldsymbol{\mu}(\mathbf{D})$ & $\mathbf{D}(\mathbf{\AA})$ & $\mathbf{Q P}(\mathbf{a u})$ & $\Delta \mathbf{E}_{\boldsymbol{e m}}^{\boldsymbol{t h}}$ & $\mathbf{f}$ & $\lambda_{\boldsymbol{e m}}^{\boldsymbol{t h}}$ \\
\hline 1 & $77 \%$ & $\mathrm{H}-4 \rightarrow \mathrm{L}$ & 3.887 & 0.001 & 319 & -2.20 & 0.751 & 0.744 & 3.236 & 0.001 & 383 \\
\hline 2 & $52 \%$ & $\mathrm{H} \rightarrow \mathrm{L}$ & 4.054 & 0.014 & 306 & 5.12 & 1.666 & 0.732 & 2.826 & 0.008 & 439 \\
\hline 3 & $47 \%$ & $\mathrm{H}-1 \rightarrow \mathrm{L}$ & 4.167 & 0.030 & 298 & 5.03 & 1.814 & 0.640 & & & \\
\hline 4 & $43 \%$ & $\mathrm{H} \rightarrow \mathrm{L}+1$ & 4.475 & 0.012 & 277 & 2.35 & 1.192 & 0.455 & & & \\
\hline 5 & $65 \%$ & $\mathrm{H}-2 \rightarrow \mathrm{L}$ & 4.768 & 0.141 & 260 & 4.45 & 1.734 & 0.607 & & & \\
\hline 6 & $52 \%$ & $\mathrm{H} \rightarrow \mathrm{L}+2$ & 4.975 & 0.158 & 249 & 3.86 & 1.585 & 0.600 & & & \\
\hline
\end{tabular}

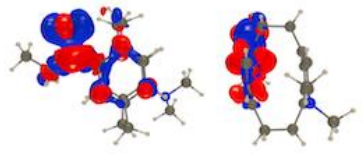

EDD $S_{1}$

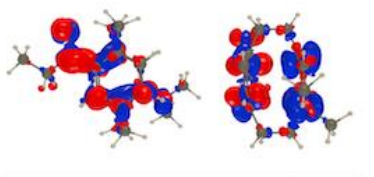

EDD $S_{2}$

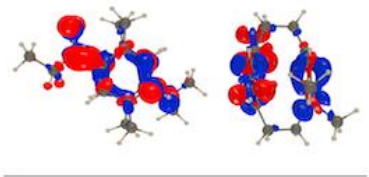

$\mathrm{EDD} \mathrm{S}_{3}$

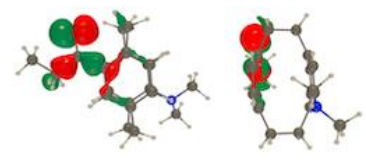

HOMO-4 (79)

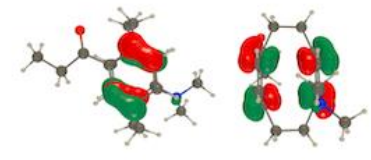

HOMO-1 (82)

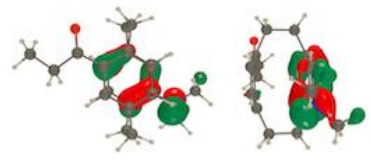

HOMO (83)

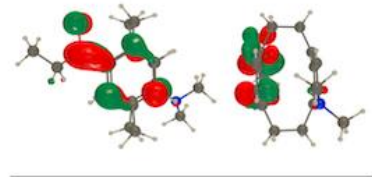

LUMO (84)

Figure S7: PCM (1,4-dioxane) - $\omega$ B97X-D/ 6-311+G(2d,p) // $\omega$ B97X-D/6-31+G(d). HOMO, LUMO and EDD plots for compound 4 in 1,4-dioxane. MO isovalue: 0.05 au \& EDD isovalue: 0.002 au. The red and blue lobes of the EDD indicate increase and decrease of the electron density upon absorption, respectively.

Table S7: PCM(Toluene) - $\omega$ B97X-D/ 6-311+G(2d,p) // $\omega$ B97X-D/6-31+G(d). Principal excited states characteristics of 4 in Toluene- Transition energies are calculated using Eq. 1

\begin{tabular}{|c|c|c|c|c|c|c|c|c|c|c|c|}
\hline $\mathbf{E S}$ & $\mathbf{\%}$ & $\mathbf{M O}$ & $\Delta \mathbf{E}_{\boldsymbol{a b s}}^{\boldsymbol{t h}}$ & $\mathbf{f}$ & $\lambda_{\boldsymbol{a b s}}^{\boldsymbol{t h}}$ & $\Delta \boldsymbol{\mu}(\mathbf{D})$ & $\mathbf{D}(\mathbf{\AA})$ & $\mathbf{Q P}(\mathbf{a u})$ & $\Delta \mathbf{E}_{\boldsymbol{e m}}^{\boldsymbol{t h}}$ & $\mathbf{f}$ & $\lambda_{\boldsymbol{e m}}^{\boldsymbol{t h}}$ \\
\hline 1 & $77 \%$ & $\mathrm{H}-4 \rightarrow \mathrm{L}$ & 3.887 & 0.001 & 319 & -2.21 & 0.756 & 0.743 & 3.238 & 0.001 & 383 \\
\hline 2 & $53 \%$ & $\mathrm{H} \rightarrow \mathrm{L}$ & 4.043 & 0.014 & 307 & 5.22 & 1.688 & 0.736 & 2.814 & 0.009 & 441 \\
\hline 3 & $48 \%$ & $\mathrm{H}-1 \rightarrow \mathrm{L}$ & 4.158 & 0.031 & 298 & 5.04 & 1.823 & 0.638 & & & \\
\hline 4 & $43 \%$ & $\mathrm{H} \rightarrow \mathrm{L}+1$ & 4.471 & 0.013 & 277 & 2.38 & 1.198 & 0.457 & & & \\
\hline 5 & $65 \%$ & $\mathrm{H}-2 \rightarrow \mathrm{L}$ & 4.758 & 0.150 & 261 & 4.47 & 1.748 & 0.604 & & & \\
\hline 6 & $52 \%$ & $\mathrm{H} \rightarrow \mathrm{L}+2$ & 4.965 & 0.161 & 250 & 3.97 & 1.607 & 0.604 & & & \\
\hline
\end{tabular}


Table S8: PCM(DCM) - $\omega$ B97X-D/ 6-311+G(2d,p) // $\omega$ B97X-D/6-31+G(d) . Principal excited states characteristics of 4 in DCM- Transition energies are calculated using Eq. 1

\begin{tabular}{|c|c|c|c|c|c|c|c|c|c|c|c|}
\hline $\mathbf{E S}$ & $\mathbf{\%}$ & $\mathbf{M O}$ & $\Delta \mathbf{E}_{\boldsymbol{a b s}}^{\boldsymbol{t h}}$ & $\mathbf{f}$ & $\lambda_{\boldsymbol{a b s}}^{\boldsymbol{t h}}$ & $\Delta \boldsymbol{\mu}(\mathbf{D})$ & $\mathbf{D}(\AA)$ & $\mathbf{Q P}(\mathbf{a u})$ & $\Delta \mathbf{E}_{\boldsymbol{e m}}^{\boldsymbol{t h}}$ & $\mathbf{f}$ & $\lambda_{\boldsymbol{e m}}^{\boldsymbol{t h}}$ \\
\hline 1 & $76 \%$ & $\mathrm{H}-4 \rightarrow \mathrm{L}$ & 3.914 & 0.002 & 317 & -2.29 & 0.804 & 0.728 & 3.219 & 0.002 & 385 \\
\hline 2 & $61 \%$ & $\mathrm{H} \rightarrow \mathrm{L}$ & 3.994 & 0.015 & 310 & 6.65 & 1.997 & 0.791 & 2.433 & 0.011 & 510 \\
\hline 3 & $56 \%$ & $\mathrm{H}-1 \rightarrow \mathrm{L}$ & 4.126 & 0.028 & 300 & 5.03 & 1.904 & 0.609 & & & \\
\hline 4 & $44 \%$ & $\mathrm{H} \rightarrow \mathrm{L}+1$ & 4.451 & 0.013 & 279 & 2.81 & 1.385 & 0.468 & & & \\
\hline 5 & $65 \%$ & $\mathrm{H}-2 \rightarrow \mathrm{L}$ & 4.725 & 0.146 & 262 & 5.17 & 2.002 & 0.607 & & & \\
\hline 6 & $52 \%$ & $\mathrm{H} \rightarrow \mathrm{L}+2$ & 4.938 & 0.163 & 251 & 4.58 & 1.848 & 0.602 & & & \\
\hline
\end{tabular}

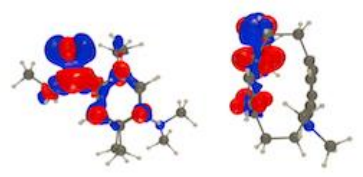

EDD $_{1}$

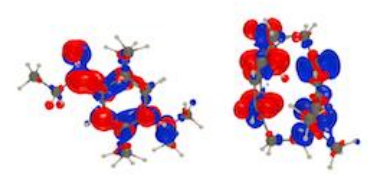

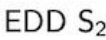

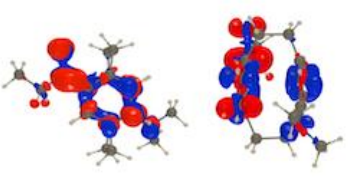

$E D D S_{3}$

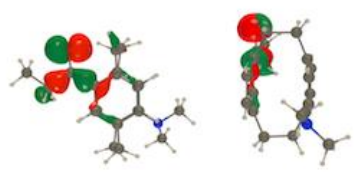

HOMO-3 (79)

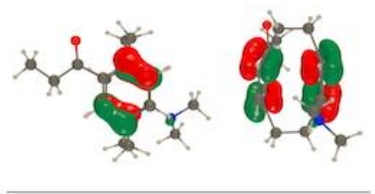

HOMO-1 (82)

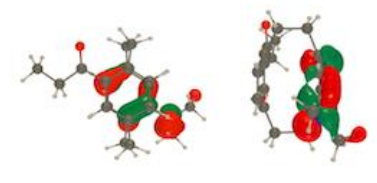

HOMO (83)

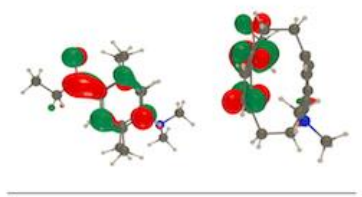

LUMO (84)

Figure S8: PCM (DCM) - $\omega$ B97X-D/ 6-311+G(2d,p) // $\omega$ B97X-D/6-31+G(d). HOMO, LUMO and EDD plots for compound 4 in DCM. MO isovalue: 0.0 au \& EDD isovalue: $0.002 \mathrm{au}$. The red and blue lobes of the EDD indicate increase and decrease of the electron density upon absorption, respectively. 

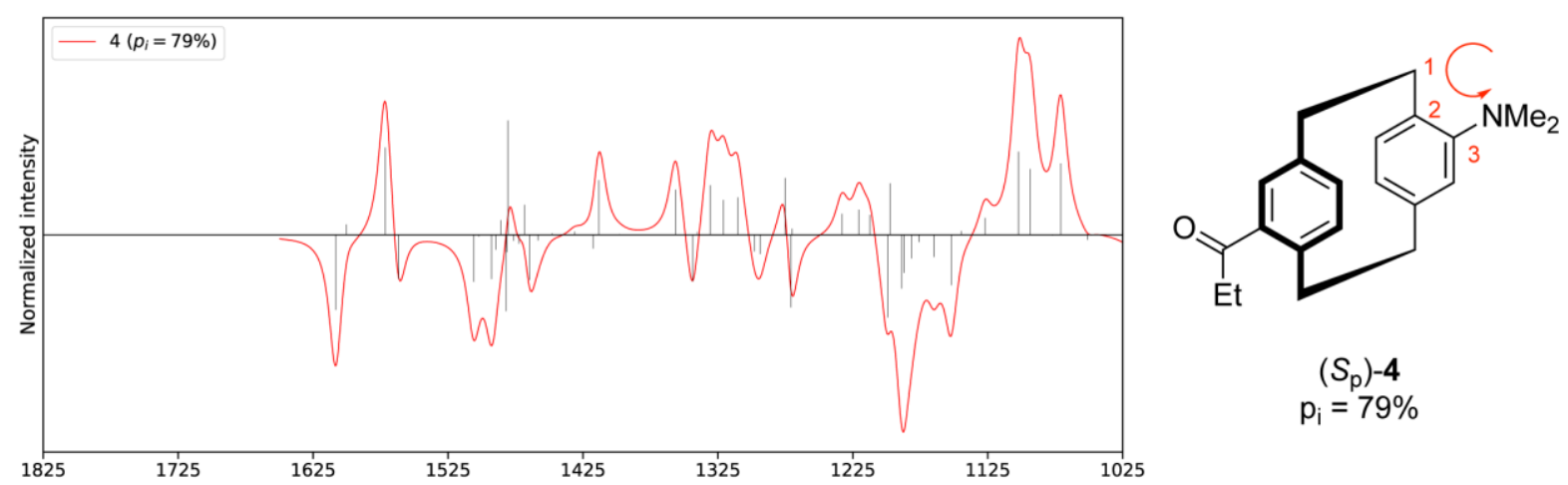

$\left(S_{p}\right)-4$

$\mathrm{p}_{\mathrm{i}}=79 \%$
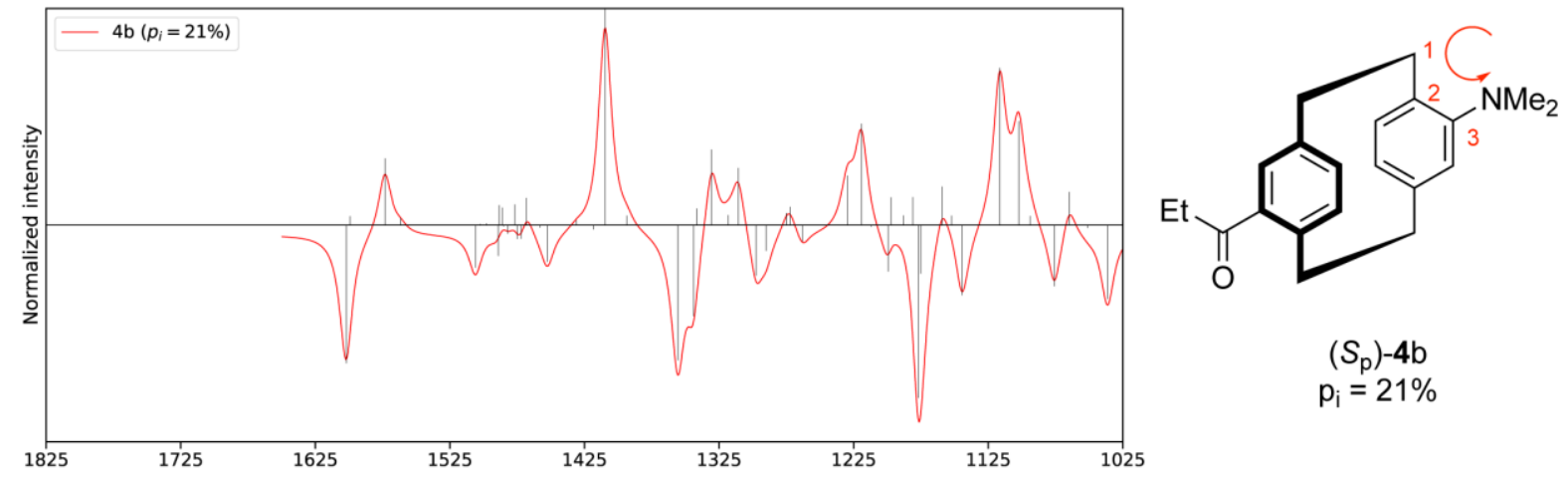

$\left(S_{\mathrm{p}}\right)-\mathbf{4 b}$

$\mathrm{p}_{\mathrm{i}}=21 \%$

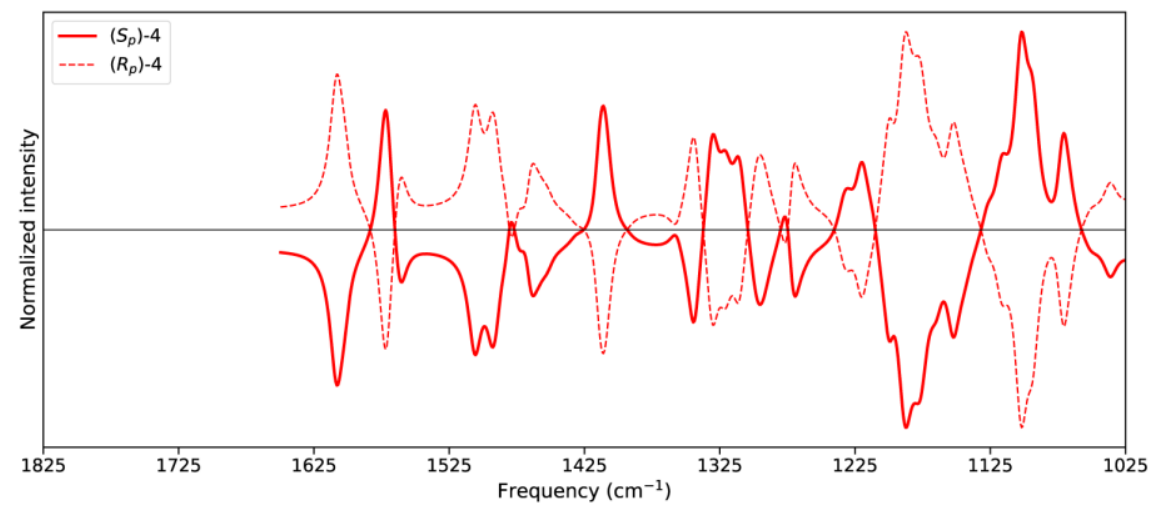

Figure S9: PCM(DCM) - B3LYP/6-31+G(d) simulated VCD for compound 4 in DCM/Frequencies have been scaled by 0.98 . Sticks have been broadened by Lorentzian functions with FWHM $=6 \mathrm{~cm}^{-1} .\left(\mathrm{S}_{\mathrm{p}}\right)-\mathbf{4}$ spectrum on the bottom is weighted by the Boltzmann population of the two conformers showed in the two top panels at $298 \mathrm{~K},\left(\mathrm{R}_{\mathrm{p}}\right)-\mathbf{4}$ spectrum is obtained mirroring $\left(\mathrm{S}_{\mathrm{p}}\right) \mathbf{- 4}$. 


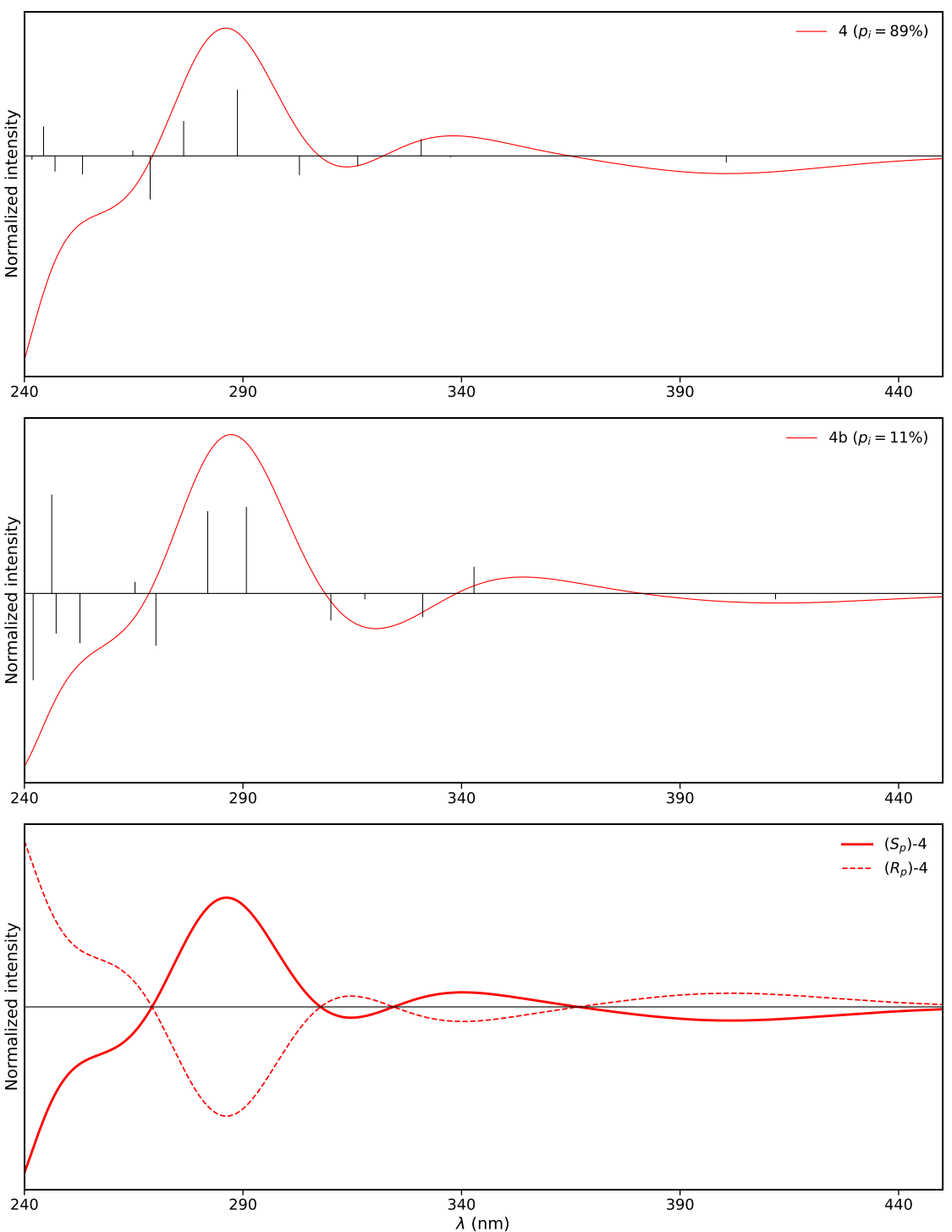

Figure S10: PCM(DCM) - B3LYP/6-311+G(2d,p) simulated ECD for compound 4 in DCM. Sticks have been broadened by Gaussian functions with FWHM $=0.25 \mathrm{eV} .\left(\mathrm{S}_{\mathrm{p}}\right)-4$ spectrum on the bottom is weighted by the Boltzmann population of the two conformers (vide supra) at $298 \mathrm{~K},\left(\mathrm{R}_{\mathrm{p}}\right)-\mathbf{4}$ spectrum is obtained mirroring $\left(\mathrm{S}_{\mathrm{p}}\right)-\mathbf{4}$. 


\section{Chiroptical properties}

IR and VCD spectra of enantiomeric pairs were recorded on a Jasco FSV-6000 spectrometer in $\mathrm{CD}_{2} \mathrm{Cl}_{2}$ in a 200 microns cell and at concentrations of $0.1 \mathrm{M}$. The half-sum of the two enantiomers has been used as the baseline for derivative 4 .

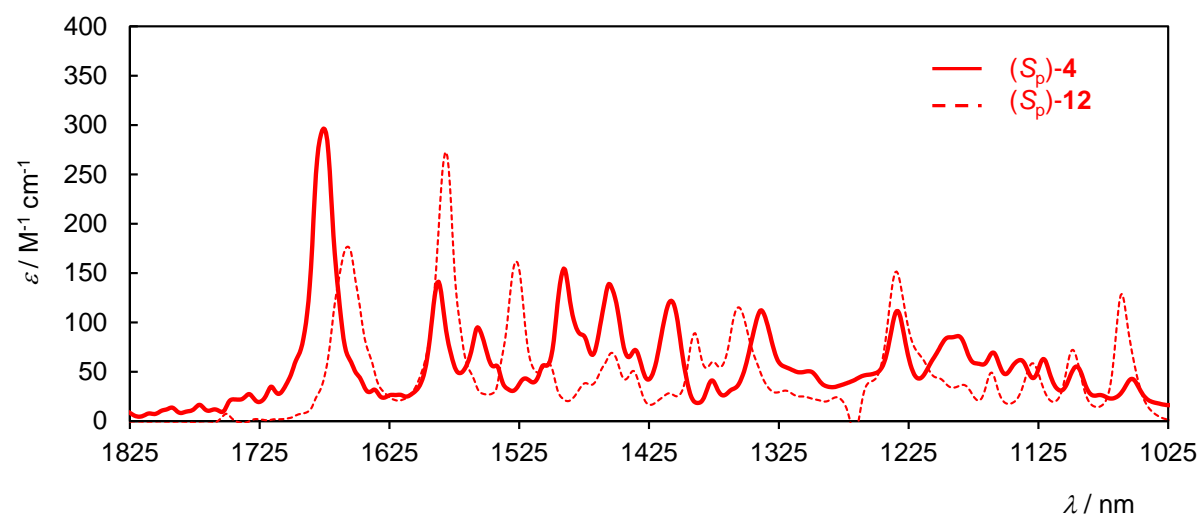

Figure S11. a) Comparison of IR spectra of $\left(S_{\mathrm{p}}\right)-\mathbf{4}$ and $\left(S_{\mathrm{p}}\right)-\mathbf{1 2}\left(\mathrm{CD} 2 \mathrm{Cl} 2,10^{-1} \mathrm{M}, 200\right.$ microns pathlength

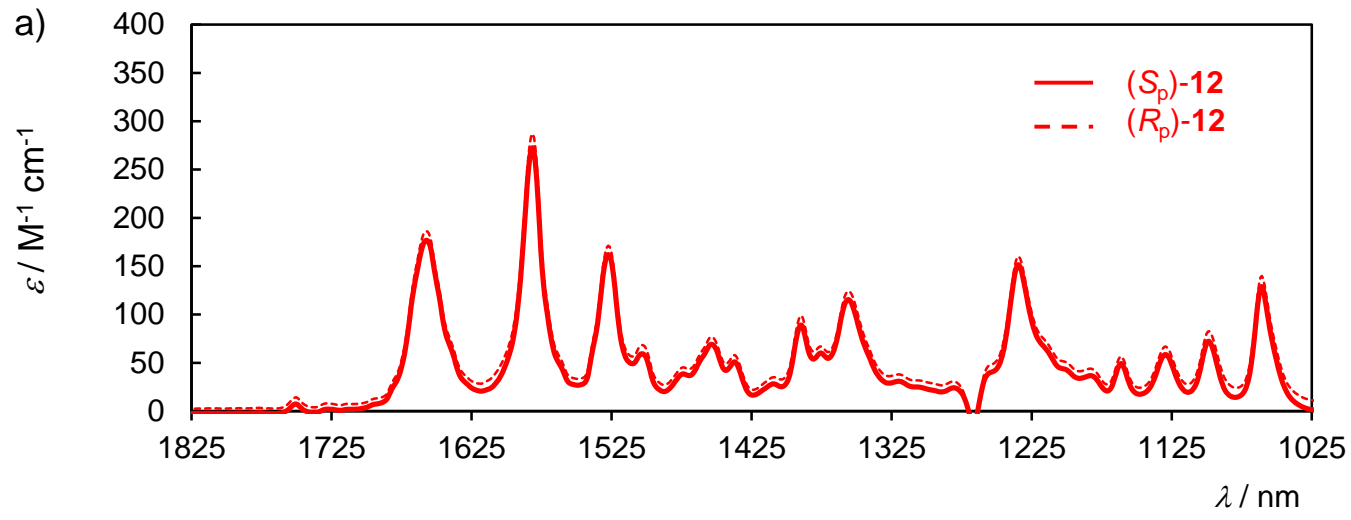

b)

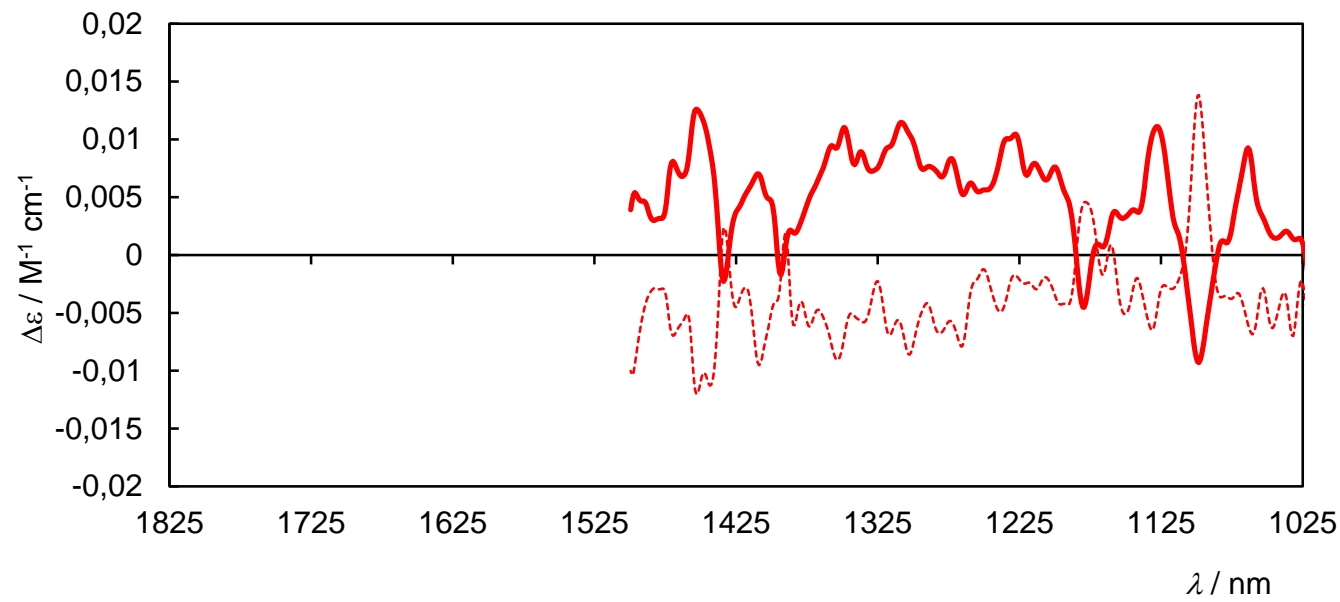

Figure S12. a) IR spectra and b) mirror-image VCD spectra of $\left(S_{\mathrm{p}}\right) /\left(R_{\mathrm{p}}\right)-\mathbf{1 2}\left(\mathrm{CD} 2 \mathrm{Cl} 2,10^{-1} \mathrm{M}, 200\right.$ microns pathlength). 
Copies of ${ }^{1} \mathrm{H}$ and ${ }^{13} \mathrm{C}$ NMR

4-Bromo-16-formyl[2.2]paracyclophane (Compound 1)

${ }^{1} \mathrm{H}$ NMR, $500 \mathrm{MHz}, \mathrm{CDCl}_{3}$

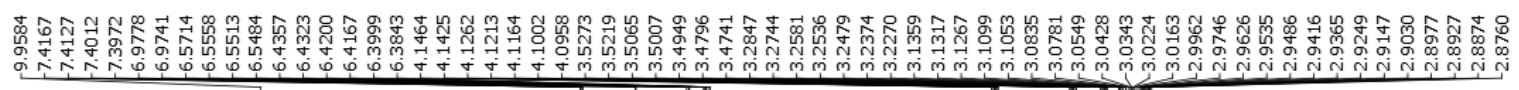
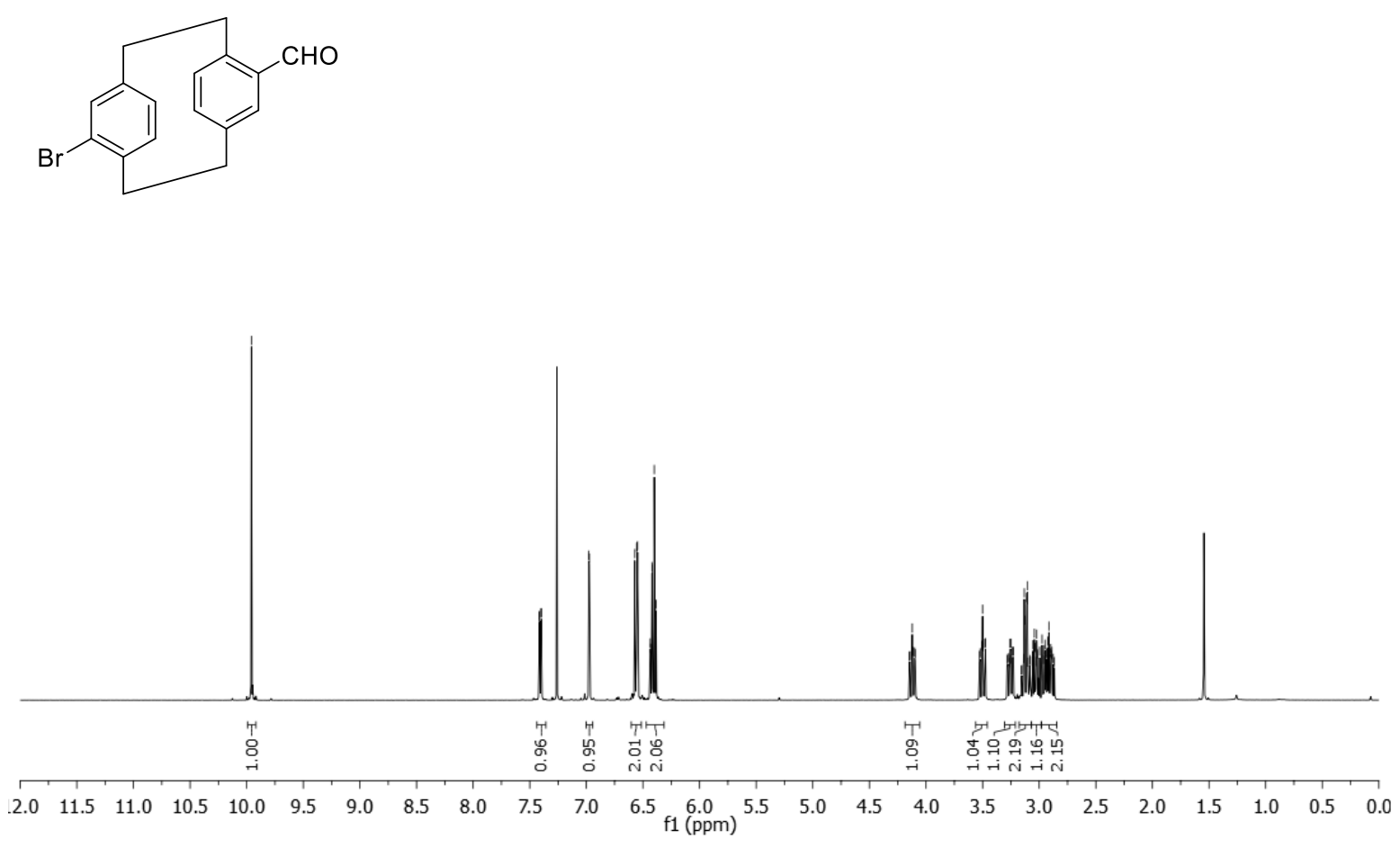

${ }^{13} \mathrm{C}\left\{{ }^{1} \mathrm{H}\right\}$ NMR, $125 \mathrm{MHz}, \mathrm{CDCl}_{3}$

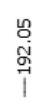

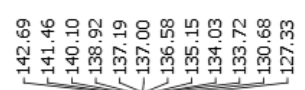

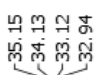
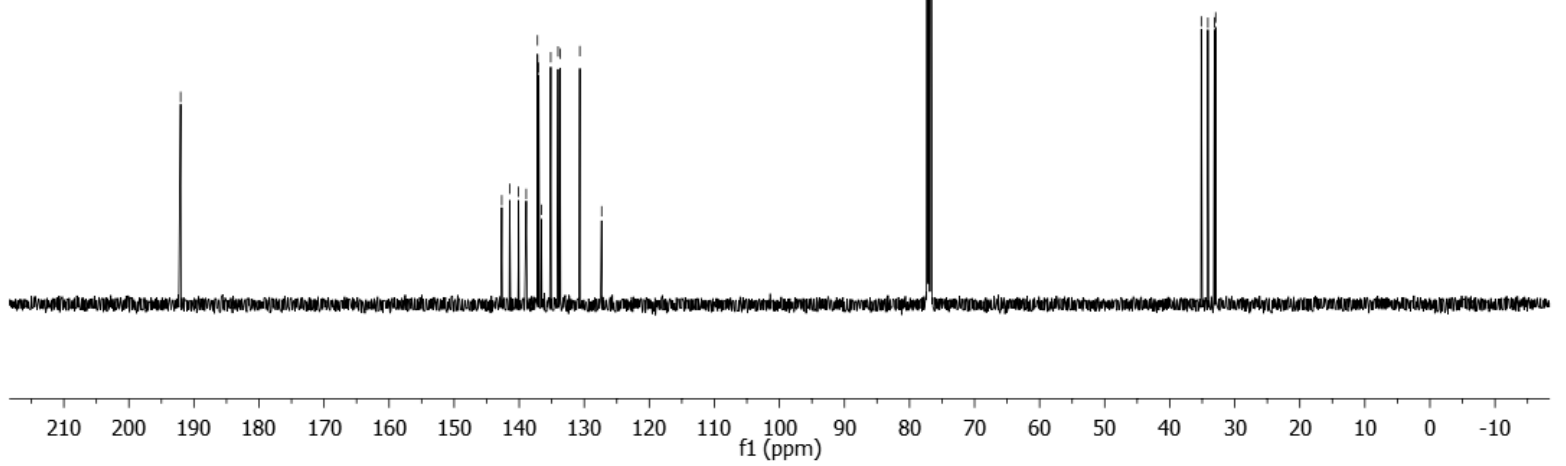

S20 
1-\{14-Bromotricyclo[8.2.2.2^\{4,7\}]hexadeca-1(12),4,6,10,13,15-hexaen-5-yl\}propan-1-ol (Compound $\mathbf{S 1}$ - major diastereosomer)

${ }^{1} \mathrm{H}$ NMR, $500 \mathrm{MHz}, \mathrm{CDCl}_{3}$

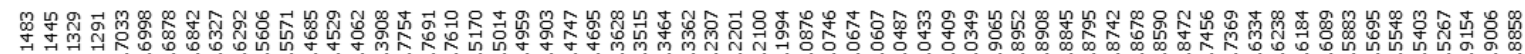

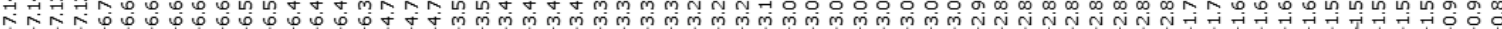<smiles>CCC(O)c1ccc2c(c1)CCc1ccc(Br)cc1CC2</smiles>

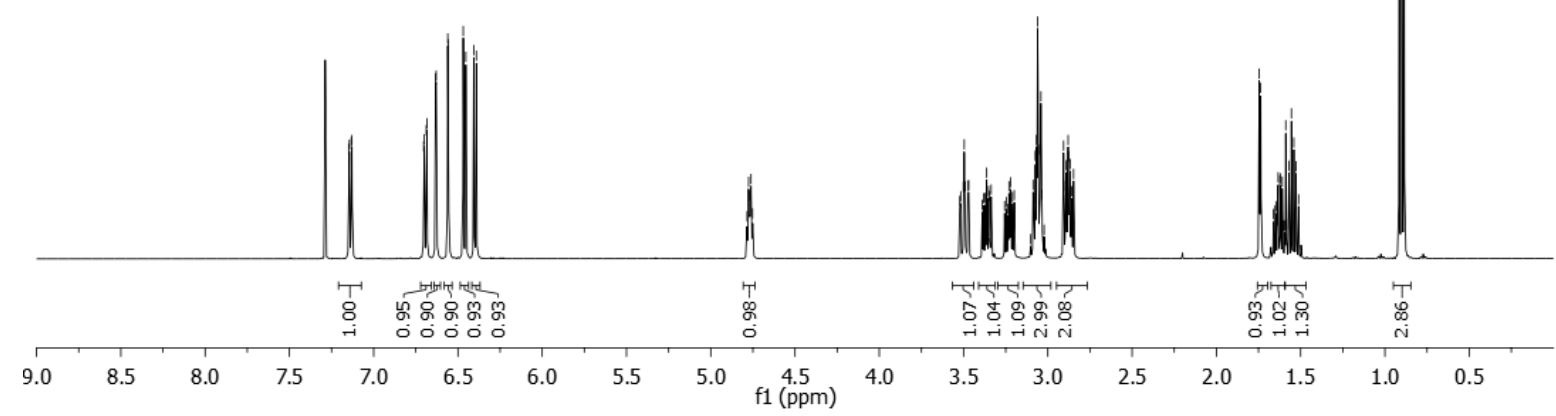

${ }^{13} \mathrm{C}\left\{{ }^{1} \mathrm{H}\right\} \mathrm{NMR}, 125 \mathrm{MHz}, \mathrm{CDCl}_{3}$

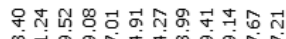

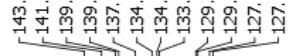


1-\{14-Bromotricyclo[8.2.2.2^\{4,7\}]hexadeca-1(12),4,6,10,13,15-hexaen-5-yl\}propan-1-one (Compound 2)

${ }^{1} \mathrm{H}$ NMR, $500 \mathrm{MHz}, \mathrm{CDCl}_{3}$

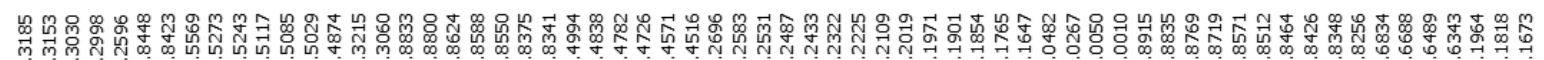<smiles>CCC(=O)c1cc2c(cc1CC)CCc1cc(Br)ccc1C2</smiles>

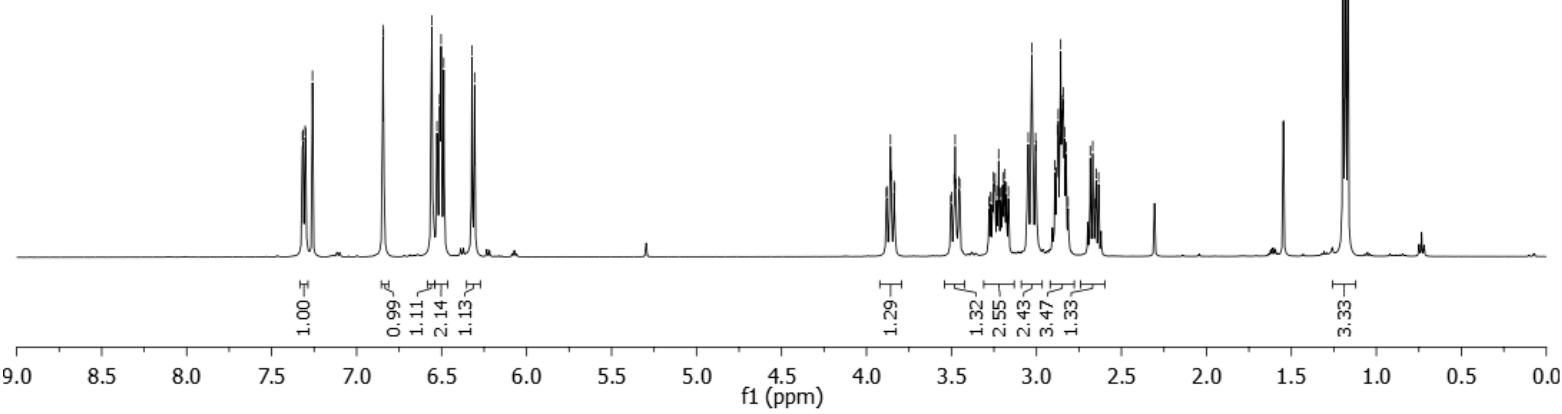

${ }^{13} \mathrm{C}\left\{{ }^{1} \mathrm{H}\right\}$ NMR, $125 \mathrm{MHz}, \mathrm{CDCl}_{3}$

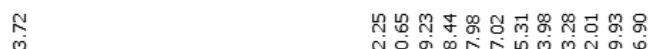

总

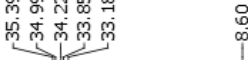

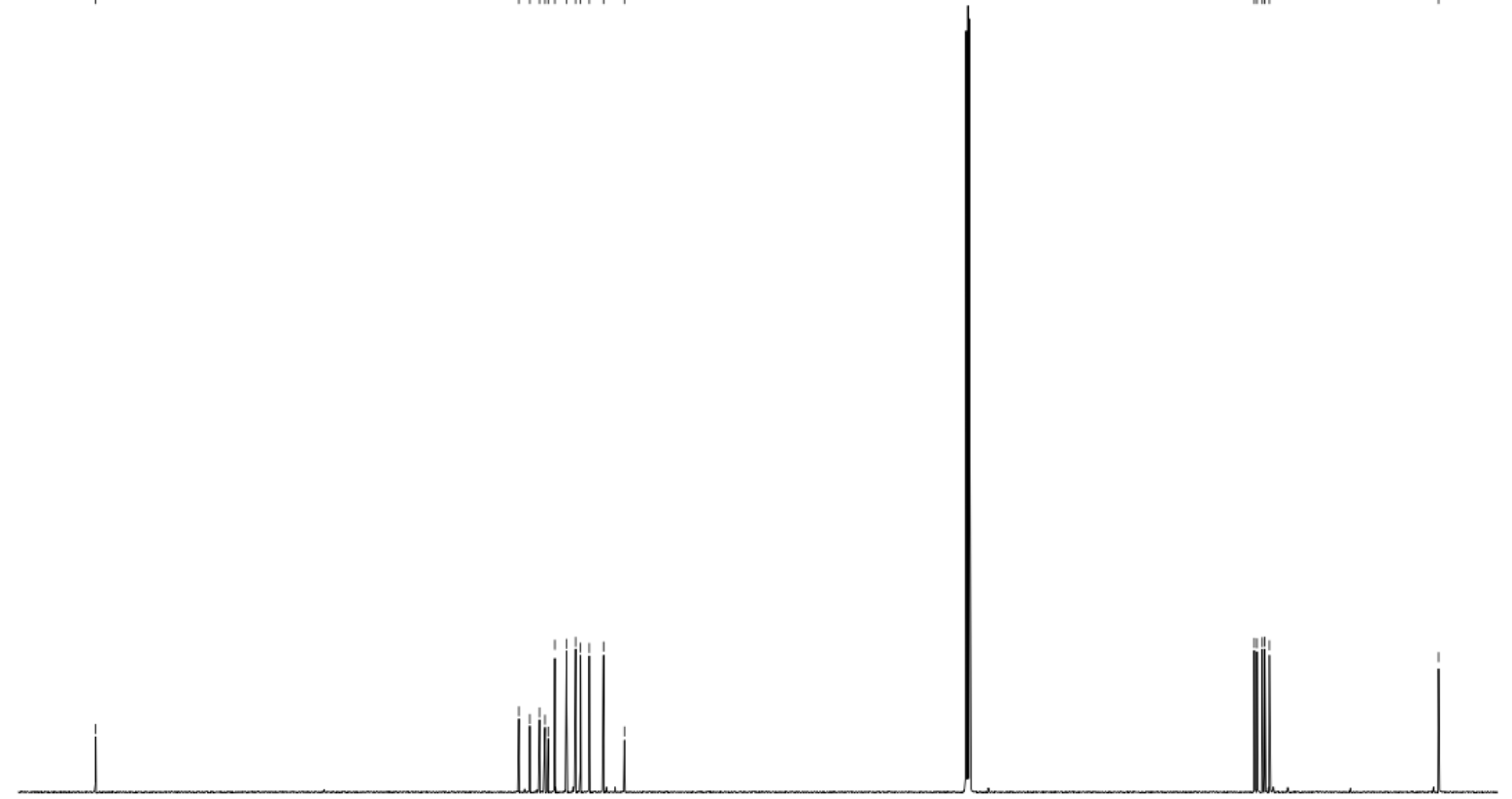

$\begin{array}{llllllllllllllllllllllllll}210 & 200 & 190 & 180 & 170 & 160 & 150 & 140 & 130 & 120 & \begin{array}{l}110 \\ \mathrm{f} 1(\mathrm{ppm})\end{array} & 100 & 90 & 80 & 70 & 60 & 50 & 40 & 30 & 20 & 10 & 0\end{array}$ 
1-\{14-Aminotricyclo[8.2.2.2^\{4,7\}]hexadeca-1(12),4,6,10,13,15-hexaen-5-yl\}propan-1-one (Compound 3)

\section{${ }^{1} \mathrm{H}$ NMR, $500 \mathrm{MHz}, \mathrm{CDCl}_{3}$}
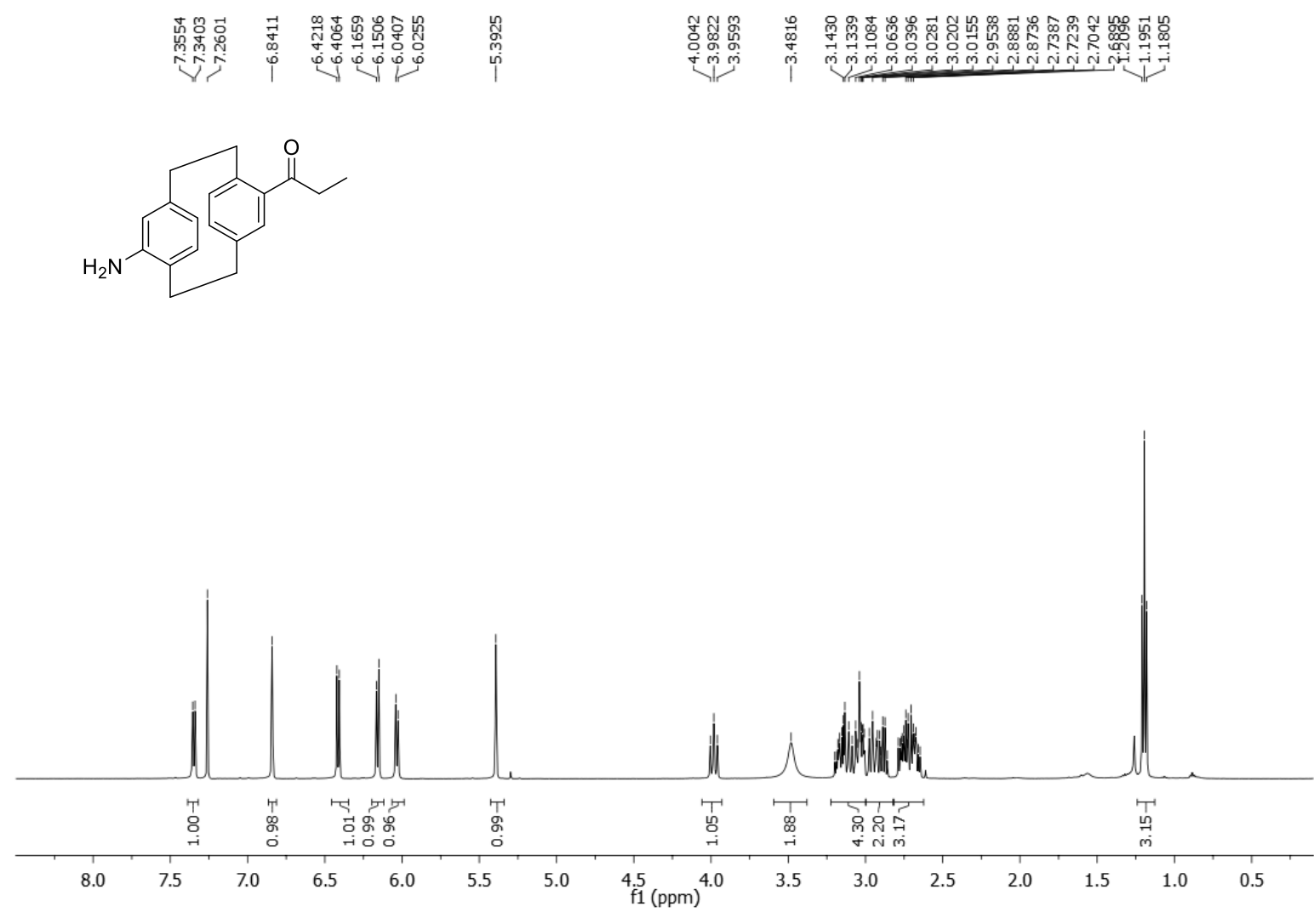

${ }^{13} \mathrm{C}\left\{{ }^{1} \mathrm{H}\right\}$ NMR, $125 \mathrm{MHz}, \mathrm{CDCl}_{3}$

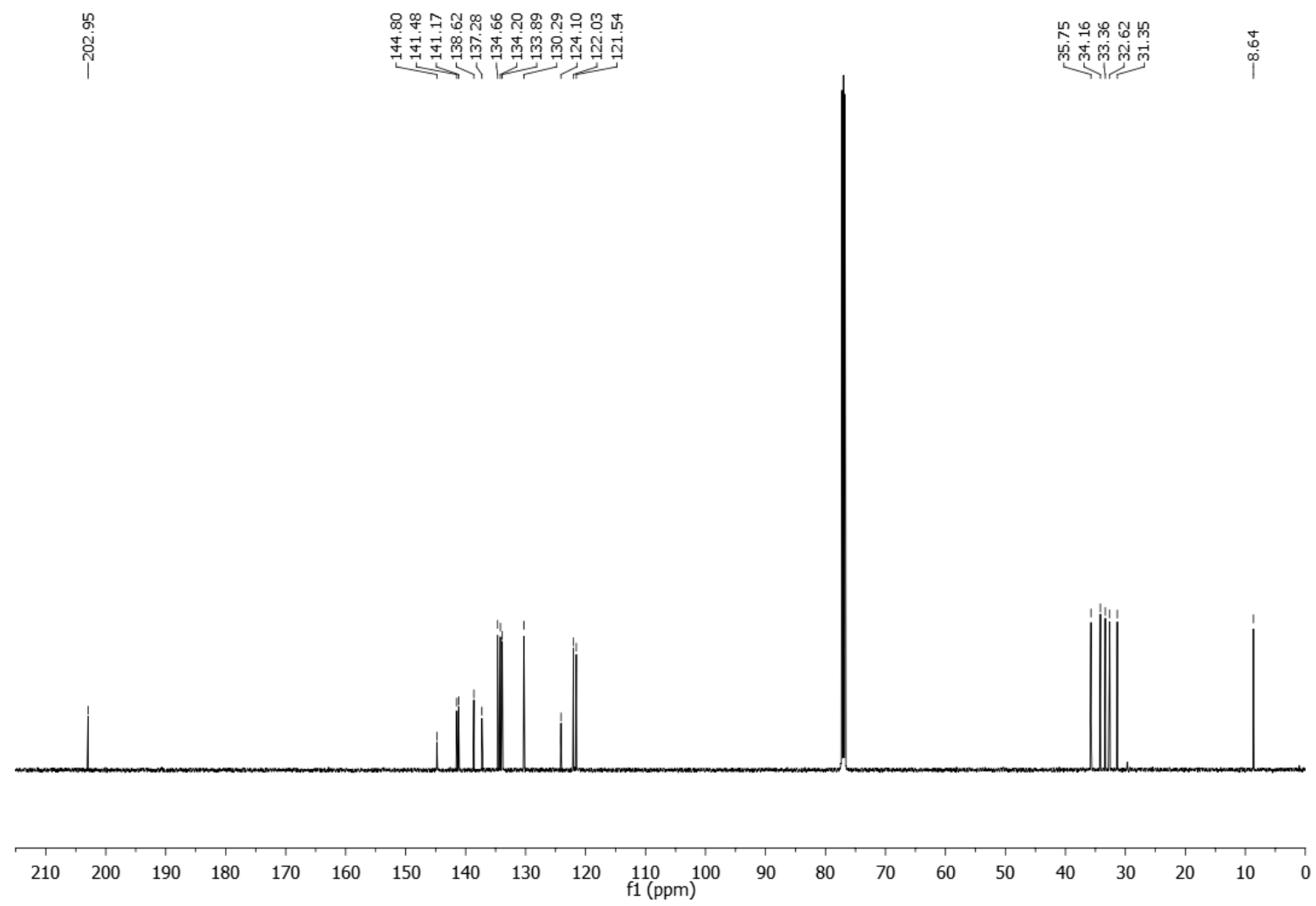


1-[14-(Dimethylamino)-tricyclo[8.2.2.2^\{4,7\}]hexadeca-1(12),4,6,10,13,15-hexaen-5-yl]propan-1one (Compound 4)

${ }^{1} \mathrm{H}$ NMR, $500 \mathrm{MHz}, \mathrm{CDCl}_{3}$

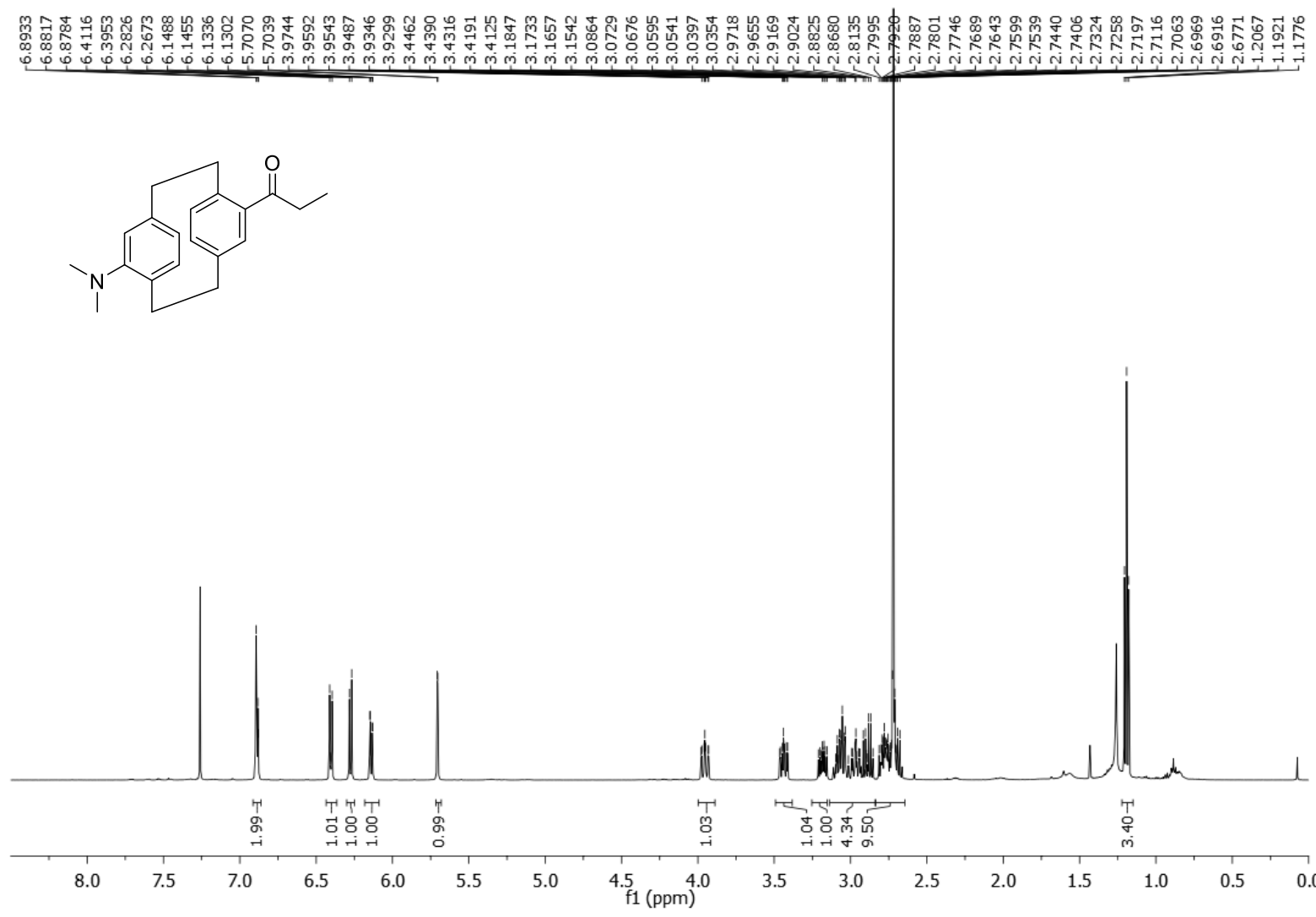

${ }^{13} \mathrm{C}\left\{{ }^{1} \mathrm{H}\right\} \mathrm{NMR}, 125 \mathrm{MHz}, \mathrm{CDCl}_{3}$

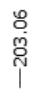

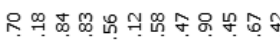

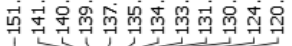

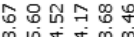

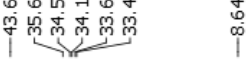

$\begin{array}{lllllllllllllllllllllll}210 & 200 & 190 & 180 & 170 & 160 & 150 & 140 & 130 & 120 & 110 & \underset{\mathrm{f} 1}{100}(\mathrm{ppm}) & 90 & 80 & 70 & 60 & 50 & 40 & 30 & 20 & 10 & 0 & -10\end{array}$ 
4,16-Diformyl[2.2]paracyclophane (Compound 5)

${ }^{1} \mathrm{H}$ NMR, $500 \mathrm{MHz}, \mathrm{CDCl}_{3}$

ईั

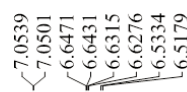

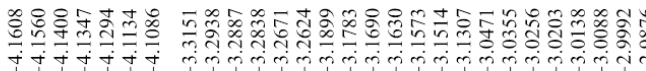

(CHO

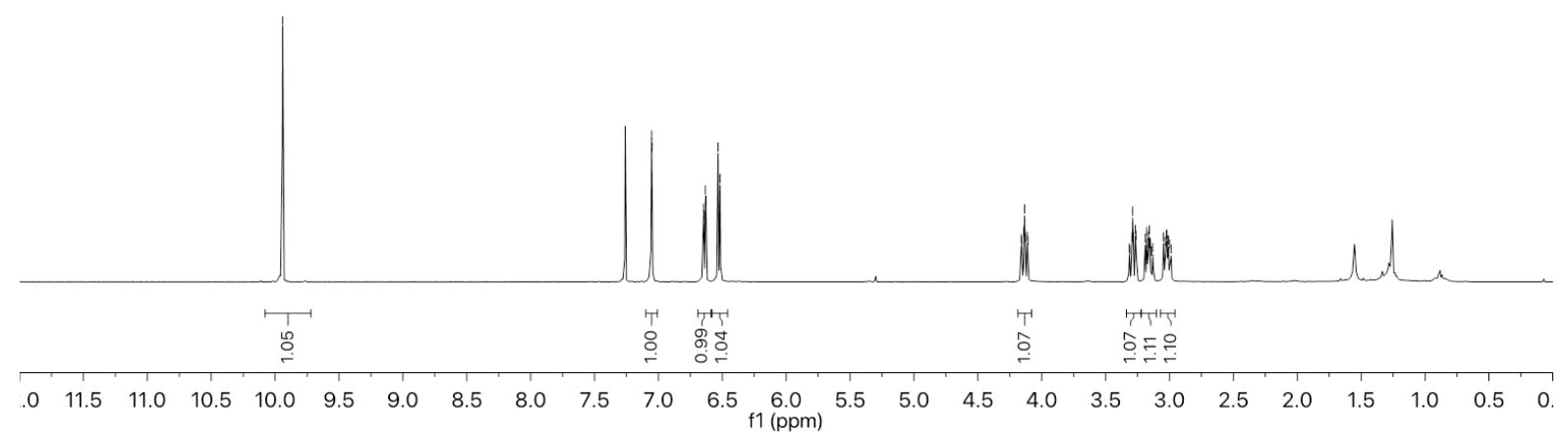

${ }^{13} \mathrm{C}\left\{{ }^{1} \mathrm{H}\right\}$ NMR, $125 \mathrm{MHz}, \mathrm{CDCl}_{3}$
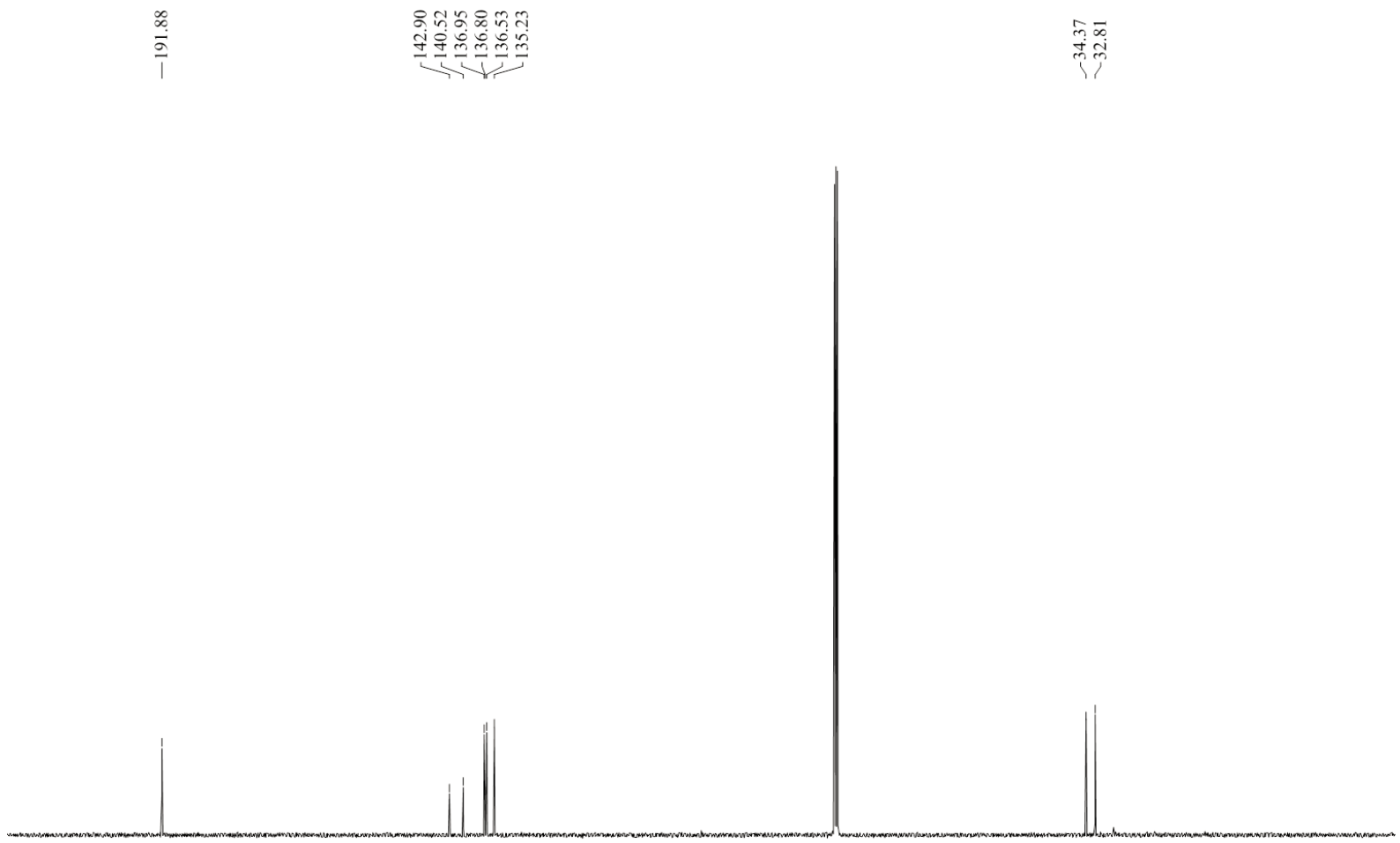

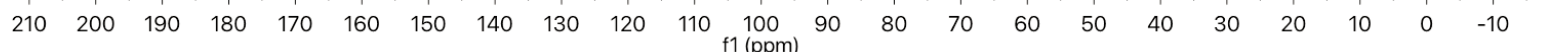


<smiles>O=Cc1cc2ccc1CCc1cccc(c1)CC2</smiles>

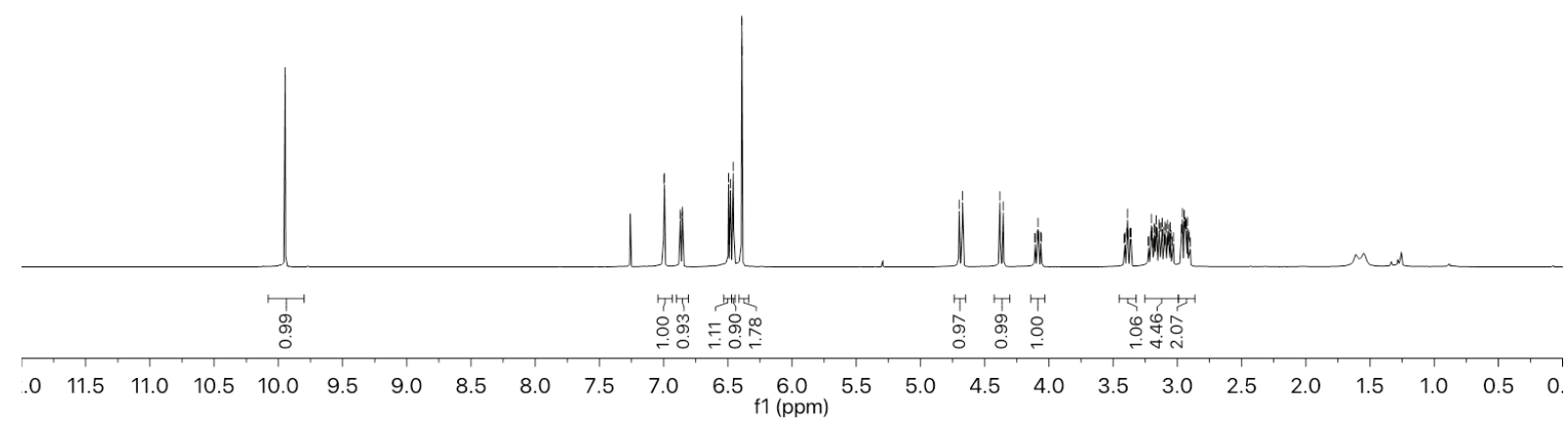

${ }^{13} \mathrm{C}\left\{{ }^{1} \mathrm{H}\right\}$ NMR, $125 \mathrm{MHz}, \mathrm{CDCl}_{3}$

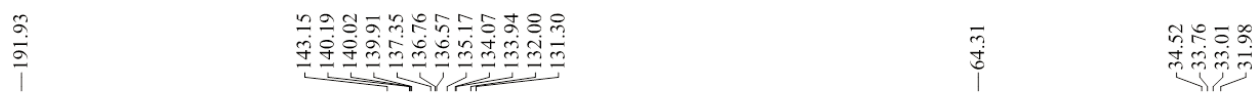

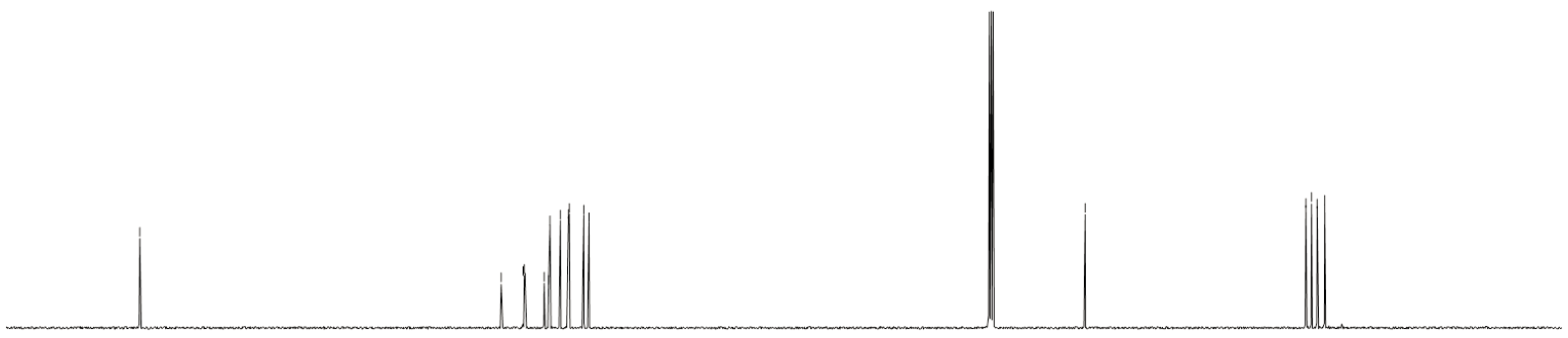

\begin{tabular}{llllllllllllllllllllllll}
\hline 1 & 200 & 190 & 180 & 170 & 160 & 150 & 140 & 130 & 120 & $\underset{1}{110}(\mathrm{ppm})$ & 100 & 90 & 80 & 70 & 60 & 50 & 40 & 30 & 20 & 10 & (
\end{tabular} 
11-Propanoyltricyclo[8.2.2.2^\{4,7\}]hexadeca-1(12),4(16),5,7(15),10,13-hexaene-5carbaldehyde (Compound 7)

${ }^{1} \mathrm{H}$ NMR, $500 \mathrm{MHz}, \mathrm{CDCl}_{3}$

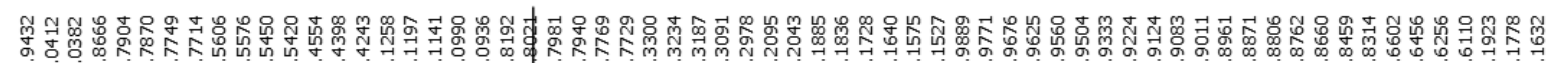

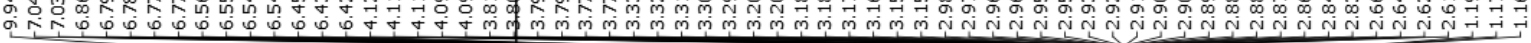<smiles></smiles>

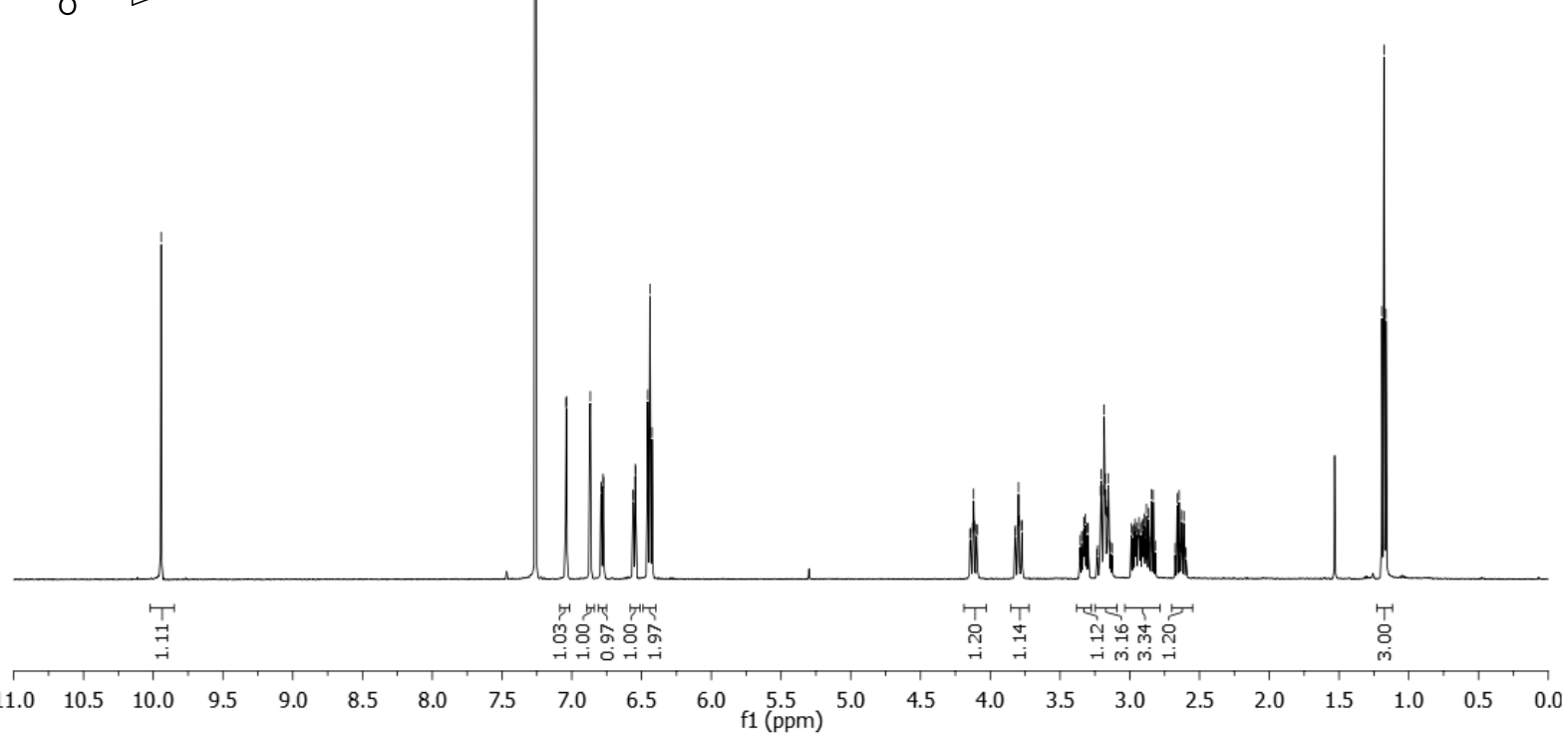

${ }^{13} \mathrm{C}\left\{{ }^{1} \mathrm{H}\right\}$ NMR, $125 \mathrm{MHz}, \mathrm{CDCl}_{3}$

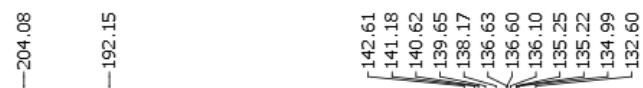


1-\{14-[(1E)-(hydroxyimino)methyl]tricyclo[8.2.2.2^\{4,7\}] hexadeca-1(12),4,6,10,13,15hexaen-5-yl)propan-1-one ((Compound 8)

${ }^{1} \mathrm{H}$ NMR, $500 \mathrm{MHz}, \mathrm{CDCl}_{3}$

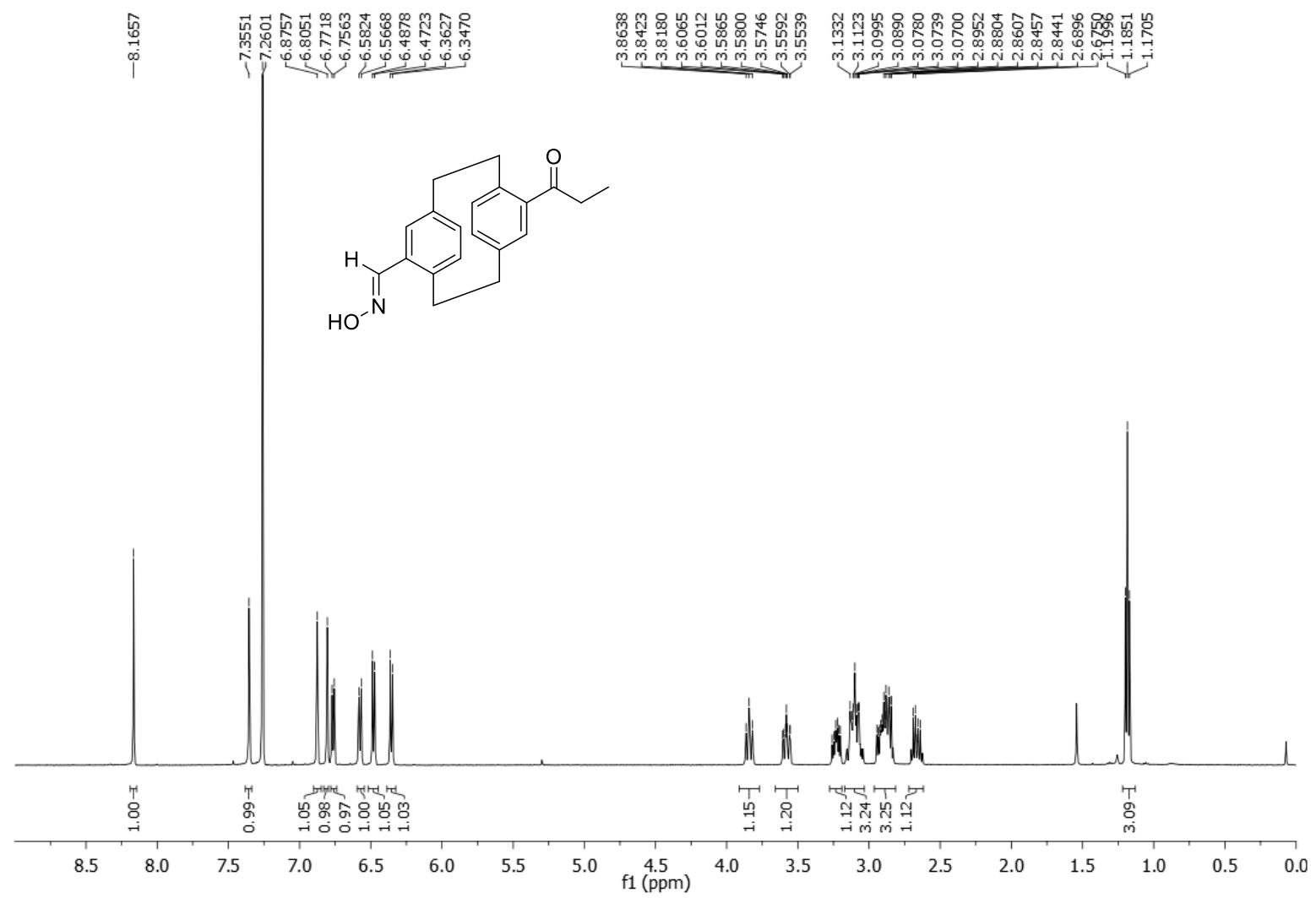

${ }^{13} \mathrm{C}\left\{{ }^{1} \mathrm{H}\right\} \mathrm{NMR}, 125 \mathrm{MHz}, \mathrm{CDCl}_{3}$

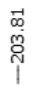

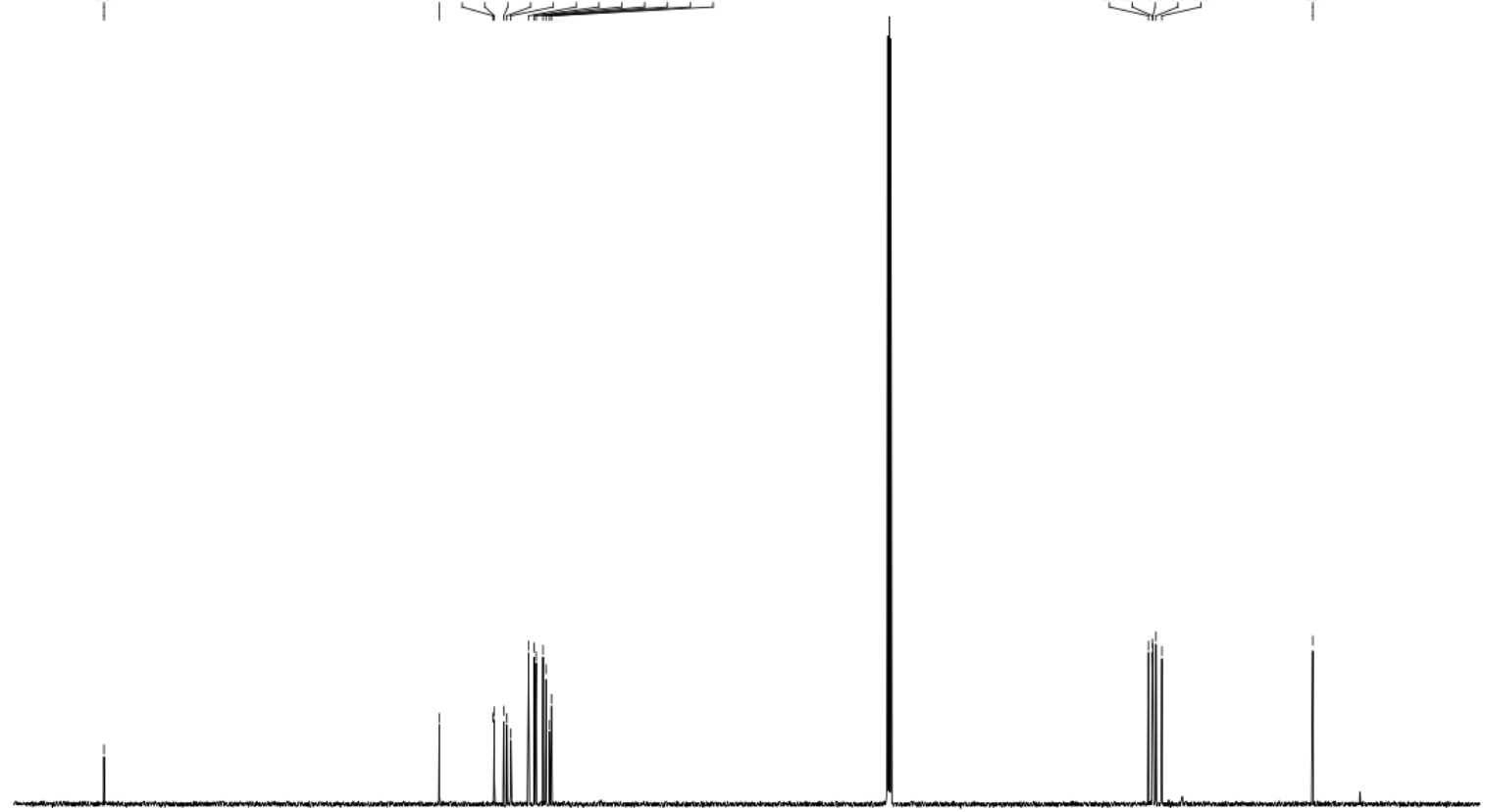

$\begin{array}{lllllllllllllllllllllll}210 & 200 & 190 & 180 & 170 & 160 & 150 & 140 & 130 & 120 & 110 & 100 & 90 & 80 & 70 & 60 & 50 & 40 & 30 & 20 & 10 & 0 & -10\end{array}$ 
4-Dimethylamino[2.2]paracyclophane (( \pm -10)

${ }^{1} \mathrm{H}$ NMR, $500 \mathrm{MHz}, \mathrm{CDCl}_{3}$

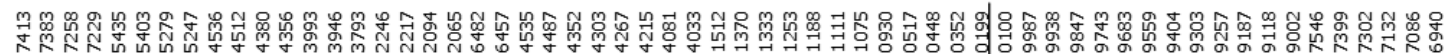

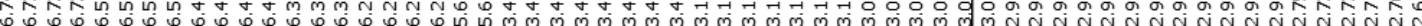

$N^{\mathrm{NMe}_{2}}$

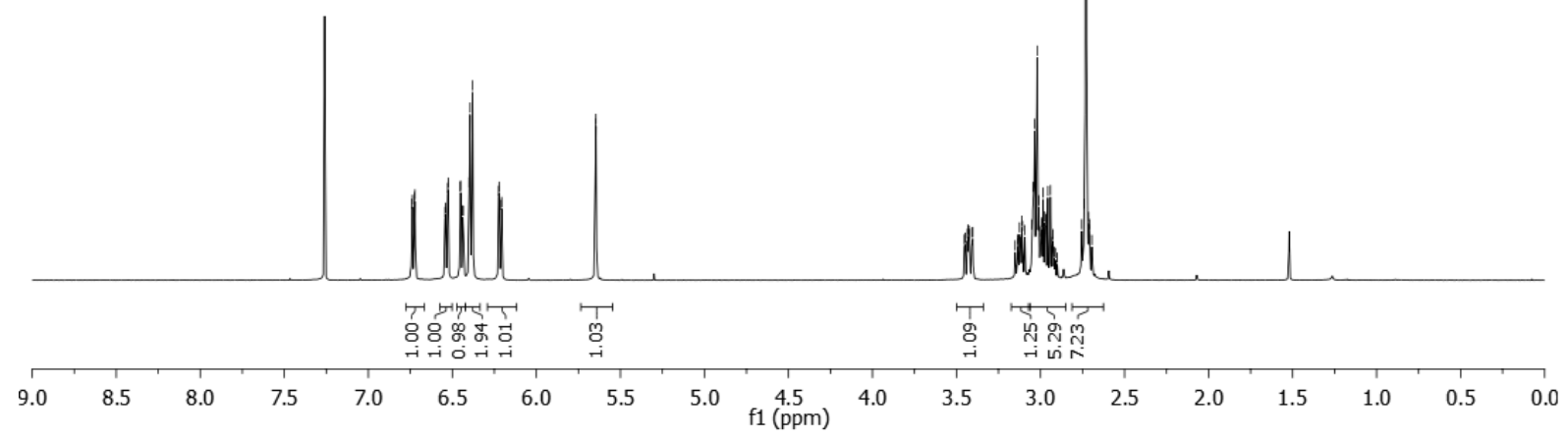

${ }^{13} \mathrm{C}\left\{{ }^{1} \mathrm{H}\right\} \mathrm{NMR}, 125 \mathrm{MHz}, \mathrm{CDCl}_{3}$

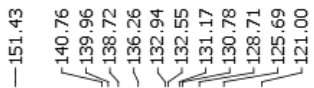

ำกำ

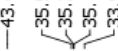

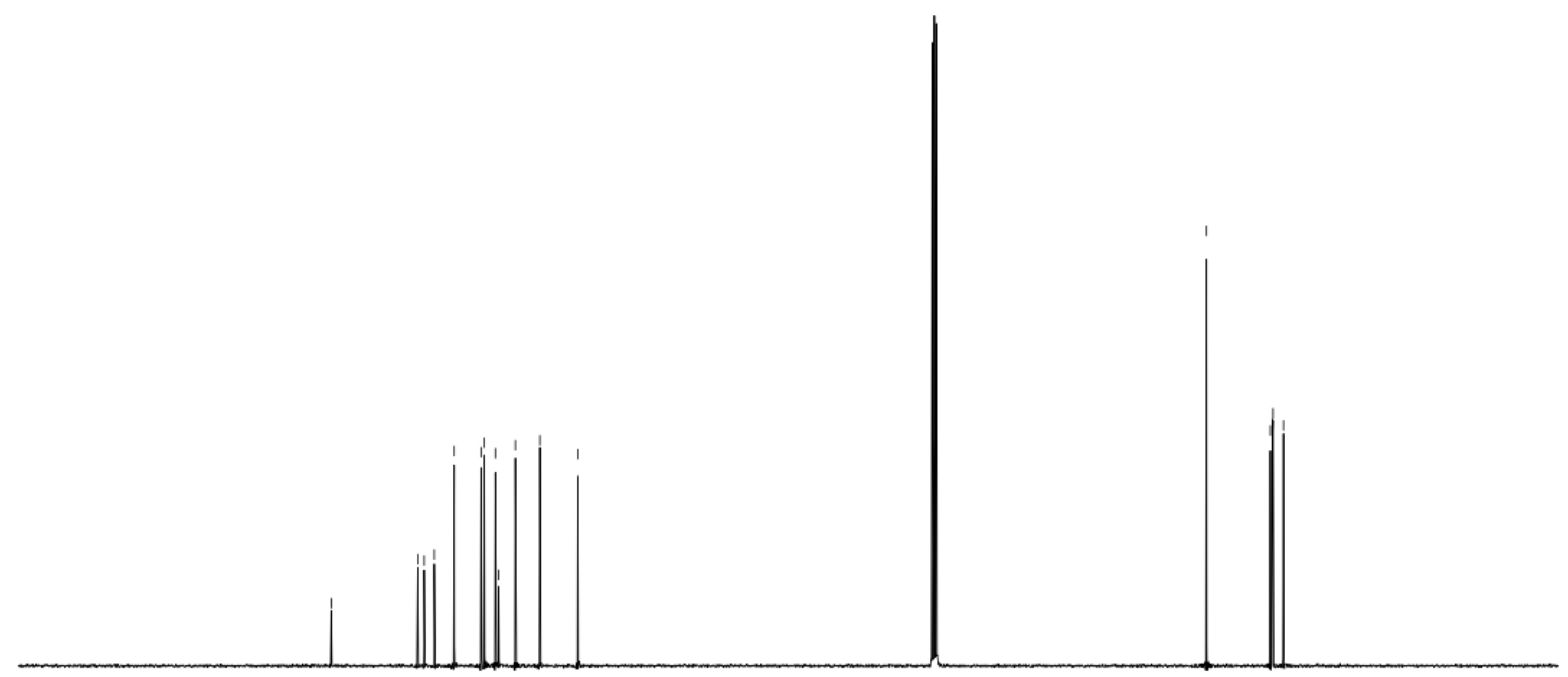

$\begin{array}{lllllllllll}190 & 180 & 170 & 160 & 150 & 140 & 130 & 120 & 110 & 100 & 90\end{array}$

$\begin{array}{lllllllll}80 & 70 & 60 & 50 & 40 & 30 & 20 & 10 & 0\end{array}$ 
4-Dimethylamino-7-formyl[2.2]paracyclophane (( \pm$)-11)$

${ }^{1} \mathrm{H}$ NMR, $500 \mathrm{MHz}, \mathrm{CDCl}_{3}$

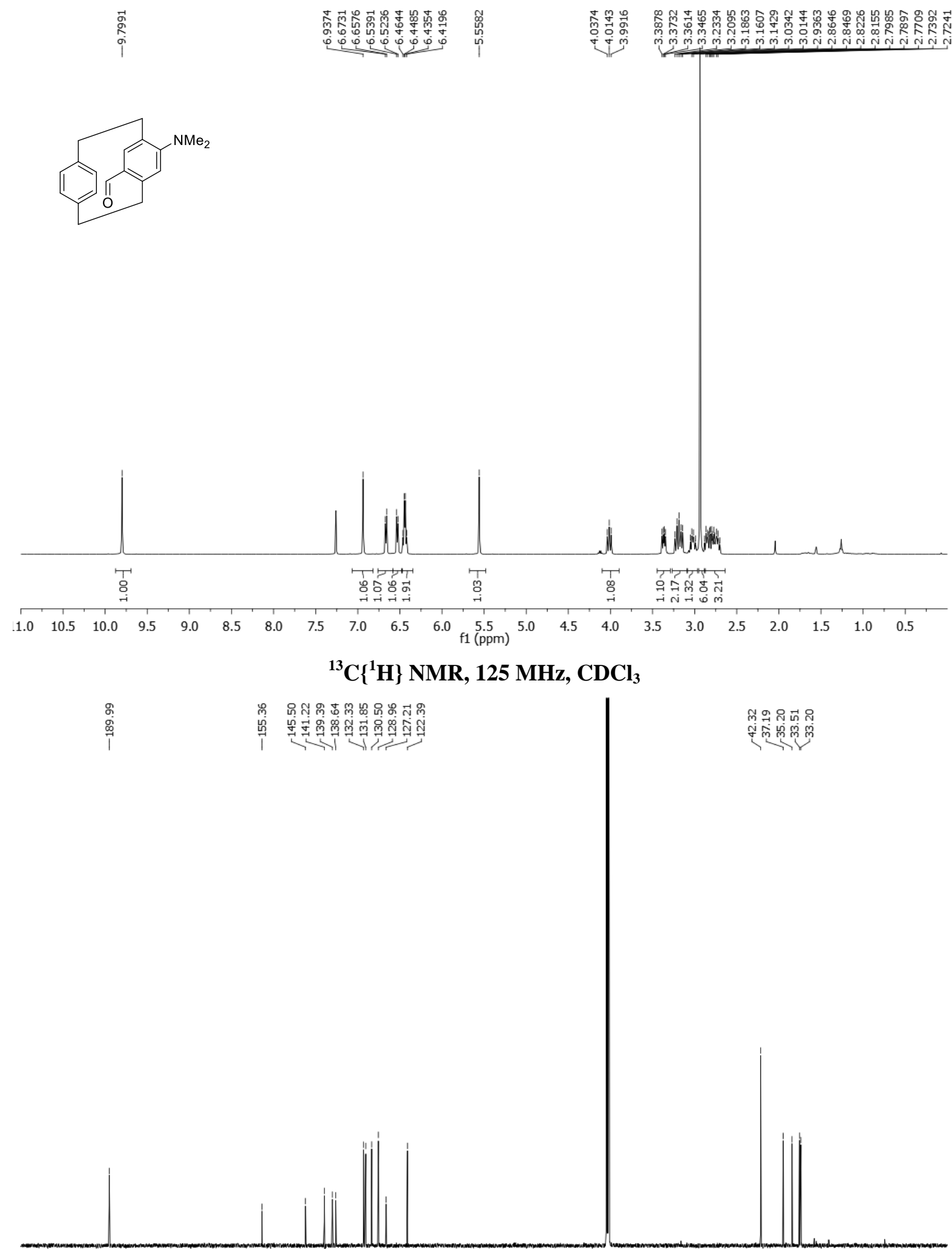

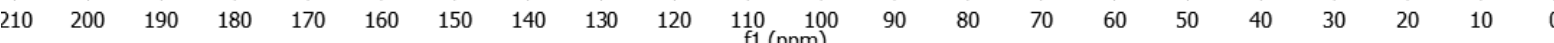


1-[15-(Dimethylamino)tricyclo[8.2.2.24, 7 hexadeca-1(12),4(16),5,7(15),10,13-hexaen-5yl]propan-1-one (( \pm$)-12)$

${ }^{1} \mathrm{H}$ NMR, $500 \mathrm{MHz}, \mathrm{CDCl}_{3}$
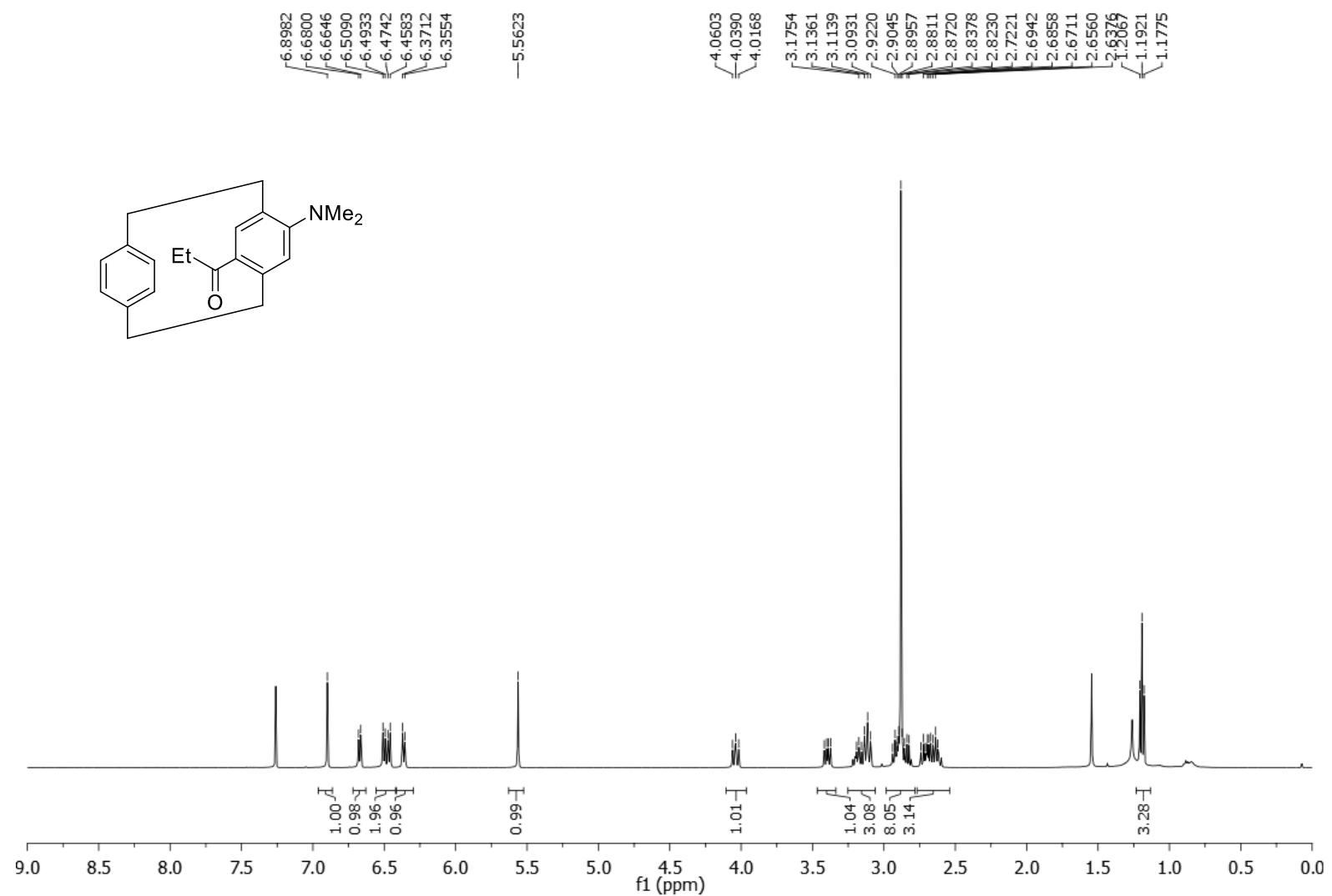

${ }^{13} \mathrm{C}\left\{{ }^{1} \mathrm{H}\right\}$ NMR, $125 \mathrm{MHz}, \mathrm{CDCl}_{3}$

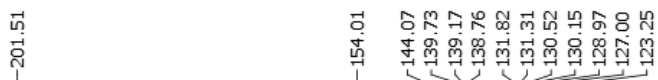

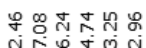

वं लिं लेंm

$\stackrel{N}{\circ}$

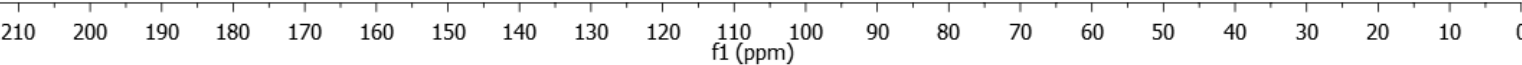

